\title{
Adjoint-Based Optimization of Displacement Ventilation Flow
}

\author{
Saleh Nabi* \\ Piyush Grover ${ }^{\dagger}$ \\ C. P. Caulfield $\ddagger$
}

July 21, 2017

\begin{abstract}
We demonstrate the use of the 'Direct-Adjoint-Looping method' for the identification of optimal buoyancy-driven ventilation flows governed by Boussinesq equations. We use the incompressible Reynoldsaveraged Navier-Stokes (RANS) equations, derive the corresponding adjoint equations and solve the resulting sensitivity equations with respect to inlet conditions. We focus on a displacement ventilation scenario with a steady plume due to a line source. Subject to an enthalpy flux constraint on the incoming flow, we identify boundary conditions leading to 'optimal' temperature distributions in the occupied zone. Our results show that depending on the scaled volume and momentum flux of the inlet flow, qualitatively different flow regimes may be obtained. The numerical optimal results agree with analytically obtained optimal inlet conditions available from classical plume theory in an 'intermediate' regime of strong stratification and two-layer flow.
\end{abstract}

Keywords Direct-Adjoint-Looping method; Displacement ventilation; Forced convection; Line plume; Intermediate regime; Numerical PDE-constrained optimization

\footnotetext{
*Mitsubishi Electric Research Labs, Cambridge, MA, USA. Corresponding Author. Email: nabi@merl.com

${ }^{\dagger}$ Mitsubishi Electric Research Labs, Cambridge, MA, USA.

$\ddagger$ BP Institute and Department of Applied Mathematics \& Theoretical Physics (DAMTP), University of Cambridge, Cambridge, CB3 0EZ, United Kingdom
} 


\section{Introduction}

\section{Nomenclature}

\begin{tabular}{|c|c|c|c|}
\hline $\mathbb{D}$ & problem domain & $\Omega$ & region of interest \\
\hline$a$ & area & $\alpha$ & entrainment coefficient \\
\hline$B, Q, W$ & $\begin{array}{l}\text { buoyancy, momentum and heat } \\
\text { flux }\end{array}$ & $\alpha^{\prime}, \beta^{\prime}$ & $\begin{array}{l}\text { non-dimensional weighting fac- } \\
\text { tor in cost function }\end{array}$ \\
\hline$C_{p}$ & specific heat & $\beta_{00}$ & $\begin{array}{l}\text { coefficient of volumetric expan- } \\
\text { sion }\end{array}$ \\
\hline$d$ & enthalpy flux representative & $\gamma$ & $\begin{array}{l}\text { weighting factor for temperature } \\
\text { in cost function }\end{array}$ \\
\hline$D$ & Universal plume constant & $\zeta$ & non-dimensional interface height \\
\hline$g$ & gravitational force & $\theta$ & non-dimensional temperature \\
\hline$G r$ & Grashof number & $\kappa$ & diffusivity \\
\hline$h$ & interface height & $\nu$ & viscosity \\
\hline$k$ & conductivity & $\rho$ & density \\
\hline$H, L$ & height and length & $\omega$ & height of the region of interest \\
\hline $\mathbf{n}, \hat{\mathbf{y}}$ & unit normal and vertical vector & $\mathcal{J}$ & cost function \\
\hline \multirow[t]{2}{*}{$P e$} & Péclet number & $\mathcal{L}$ & augmented cost function \\
\hline & & $\mathcal{P}, \mathcal{W}, \mathcal{U}$ & $\begin{array}{l}\text { vector of adjoint, direct and de- } \\
\text { sign variables }\end{array}$ \\
\hline$q^{\prime \prime}$ & heat flux per length & Subscripts & \\
\hline$R e$ & Reynolds number & $a$ & adjoint \\
\hline$R i$ & Richardson number & $d$ & desired \\
\hline$T, V, p$ & $\begin{array}{l}\text { temperature, velocity and pres- } \\
\text { sure fields }\end{array}$ & eff & effective \\
\hline$V_{r}$ & velocity ratio & in & inlet \\
\hline$T_{\text {comf }}$ & comfortable temperature & $\mathrm{p}$ & plume \\
\hline$x$ & horizontal coordination & ref & reference value \\
\hline \multirow[t]{2}{*}{$y$} & vertical coordination & Superscripts & \\
\hline & & $\mathrm{O}$ & optimal \\
\hline
\end{tabular}

Modern buildings contribute close to $40 \%$ of total energy consumption in North America, a large fraction of which is used in HVAC [1]. Design of indoor environments has the twin goals of providing optimal thermal comfort while minimizing energy consumption. The complicated dynamics of airflow within the built environment, and its interaction with occupants, electrical devices and the exterior, necessitate development of a systematic approach to fulfill the requirements of such a design.

There are various methods to determine air velocity, temperature, relative humidity, and contaminant concentration in a room, such as computational fluid dynamics (CFD), analytical models, and experimental measurements 2]. The methods help to identify flow regimes with the desired air quality, thermal comfort, and energy consumption. By using such methods in a trial-and-error process, engineers have been able to 
design HVAC systems to achieve desired conditions in the built environment [3]. However, this process may involve a large number of iterations and rely heavily on a good initial guess of conditions, which requires prior knowledge and experience. 'Moreover, there is no guarantee that the final solution is even close to optimal, in terms of meeting the design objective, and no physical insight is obtained using a trial-and-error process. The formal definition of optimal solution is given in Section 4 .

An efficient determination of the design of an HVAC system for a desirable built environment requires a systematic approach that starts with the design objective. The design objective can be expressed in terms of field variables, e.g. air velocity or temperature, representing thermal comfort, energy consumption, air quality, etc, in the whole or a part of the domain in transient or steady state. Recently, there have been several studies that formulate such problems as inverse design problems [3. Inverse design problems can be further classified as either 'backward' or 'forward' methods 3 . Although they have the attraction of being computationally tractable, backward methods such as the quasi-reversibility method [4, the pseudoreversibility method [5] and the regularized inverse matrix method [6], all suffer from the fact that the flow field needs to be known prior to the solution. On the other hand, forward methods, such as CFD-based adjoint methods [7, reduced-order models (ROMs) [8, 9], and genetic algorithm (GA) [10, solve for both the flow field and the scalar field, and hence have broader applications.

Reduced-order models have also been extensively used to reduce the computational burden in optimization and control applications 11. These methods rely on finding appropriate sets of dominant modes in simulation or experimental data. The problem dimension can then be reduced to the number of modes retained in the process. Genetic algorithm methods, with either a single objective or multiple objectives, are conversely much more computationally expensive 3 . Such computational cost can grow even larger if several inputs have also to be determined. However, it is important to remember that the main advantage of GA methods is that they provide global optimal solutions [10.

In the last two decades, there has been a large amount of research in the area of design optimization of fluid dynamics processes using adjoint-based methods, including shape optimization of airfoils [12, automotive design [13, wind turbines [14, and ducted flows [15. Another related recent development has been the use of nonlinear adjoint optimization techniques to find 'optimal', i.e. minimal energy, perturbations that lead to turbulence in pipe and channel flows [16, 17, 18. However, application of systematic optimization and control to indoor flows has been missing until very recently [19, 20, 21, 22, 17.

In contrast to shape optimization problems in aerodynamics, indoor airflow dynamics optimization is aimed at obtaining optimal boundary actuation that leads to desired airflow temperature and velocity distribution characteristics in the domain of interest 23. Since the flow is usually in the turbulent regime, numerical optimization using Direct Numerical Simulation (DNS) or Large Eddy Simulation (LES) is not generally feasible. This requires the use of Reynolds-Averaged Navier-Stokes (RANS) models, or some other closure models to account for interaction between the mean-flow and turbulent eddies. Since the accuracy of RANS models in indoor flow can vary according to the flow conditions, it is crucial that the optimization results are verified by direct comparison with experimental results, or with previously experimentally-validated analytical models of indoor airflow phenomenon. One such class of analytical models are physics-based reduced-order models for indoor ventilation dynamics 24. Beginning with seminal work by Baines and Turner [25, there have been several advances in our understanding of mixing and transport in indoor airflows. Experimentally validated low-order models have been developed for steady [26] and unsteady [27] natural ventilation, ventilation in the presence of time-varying heat sources 28, 29], exchange flow between adjacent rooms [30, and general unsteady plume dynamics [31], among other phenomena.

In this paper, we formulate and solve a model test-case problem to determine inlet velocity and temperature that optimize a certain cost functional related to maintaining desired temperature distribution in part of a room, using and demonstrating the utility of the so-called Direct-Adjoint-Looping (DAL) method [16. The study focuses on the fully turbulent mixed-convection regime, resulting from the presence of a line heat source in addition to forced conditioned air from the inlet. The open source software Open Field Operation and Manipulation (OpenFOAM) is used as the numerical tool to develop our continuous-adjoint based framework.

While we perform validation of forward simulation (CFD) results by comparing with experimentally 
validated models (as is customary), the focus of our work is actually on implementation of RANS-based DAL optimization on buoyancy-driven flows. In that regard, we validate our optimization results, which is an important contribution of this study. Most previous adjoint-based studies have focused on inverse design, and involved synthetic problems where the optimal solution is known a priori, and hence the validation is straightforward. In our study, the optimal solution is not known a priori, and we validate our numerical optimal solutions by comparing them to analytically obtained optimal solutions in a flow regime where the analytical (forward) model is known to be valid. Another novelty of this work lies in the inclusion of enthalpy constraints on the inlet flow in the optimization problem to better model real-life scenarios. The constrained optimization leads to nontrivial modification of optimal solutions as compared to the unconstrained case. This work is a first step towards developing a validated numerical framework for indoor flow optimization and it lays out a strategy for dealing with more realistic situations.

The rest of the paper is organized as follows. In Section 2 we derive an analytical model for the buoyancy-driven flow with bottom and top vents in the presence of a line heat source of buoyancy, building upon the existing experimentally-validated models for thermal plumes [32]. In Section 3, we study the system using RANS simulations, compare the results to the predictions of analytical models, and identify the regime of validity of those analytical models based on relevant non-dimensional parameters. In Section 4. we formulate the optimization problem, and employ the DAL method to solve the problem numerically. Analytical optimization of the reduced-order analytical model derived in Section 2 is also carried out. In Section 5, detailed comparison of the results from numerical and analytical optimization methods is carried out. We discuss the validity of the RANS based optimization, and the effect of constraints on the optimal solution. Finally in Section 6, we provide conclusions and sketch out directions for future research.

\section{Analytical Model For Displacement Ventilation}

The schematic of the model test case problem for the domain $\mathbb{D}$ is shown in Fig. 1. The height of the room is $H$, the inlet area (divided by the width of the room) is $a_{i n}$, and the source buoyancy flux is $B$, which is related to the heat flux by

$$
B=\frac{W \beta_{00} g}{\rho C_{p}}
$$

where $g$ is the acceleration due to gravity, $W$ is the heat flux, $\rho$ is the fluid density, $C_{p}$ is the fluid's specific heat capacity and $\beta_{00}$ is the coefficient of volumetric expansion (for ideal gases $\beta_{00} \approx 1 / T$ ).

There is one major difference between this setting and the traditional displacement flow for which ventilation occurs when the dense fluid enters at the bottom and displaces the lighter fluid within the space which in turn flows out through the openings at the top [26, 33. We assume that the space, for simplicity chosen to be a two-dimensional cavity with bottom and top vents, is subjected to a prescribed inflow provided by an air conditioning unit or a mechanical machine. This is in contrast with earlier studies, where the space is connected to an exterior, which in turn is modeled as a large reservoir with fixed temperature, and the inflows and outflows are driven dynamically by (assumed hydrostatic) pressure differences associated with density differences between the interior and exterior fluids. Therefore, the inlet temperature and velocity, $\left(T_{i n}, V_{i n}\right)$ are design variables for our problem. Equivalently, the inlet volume flux is determined by the design requirements instead of being a function of the temperature difference between the interior and exterior. We are most interested in the range of parameters that lead to a stable strong stratification and an interface between top and bottom layers. We explore such a flow configuration in more detail in the following.

The analytical model is intended to capture the effect of a continuous source of buoyancy (or heat) on temperature stratification. We restrict our attention to isolated sources of buoyancy, and we focus for computational convenience on a two-dimensional line plume. Extension to an axisymmetric point source is straightforward. Using integral methods and the entrainment hypothesis of Taylor, Morton et al. 32] derived equations for a buoyant plume in stationary unstratified ambient atmosphere. The volume flux within the plume is given by 


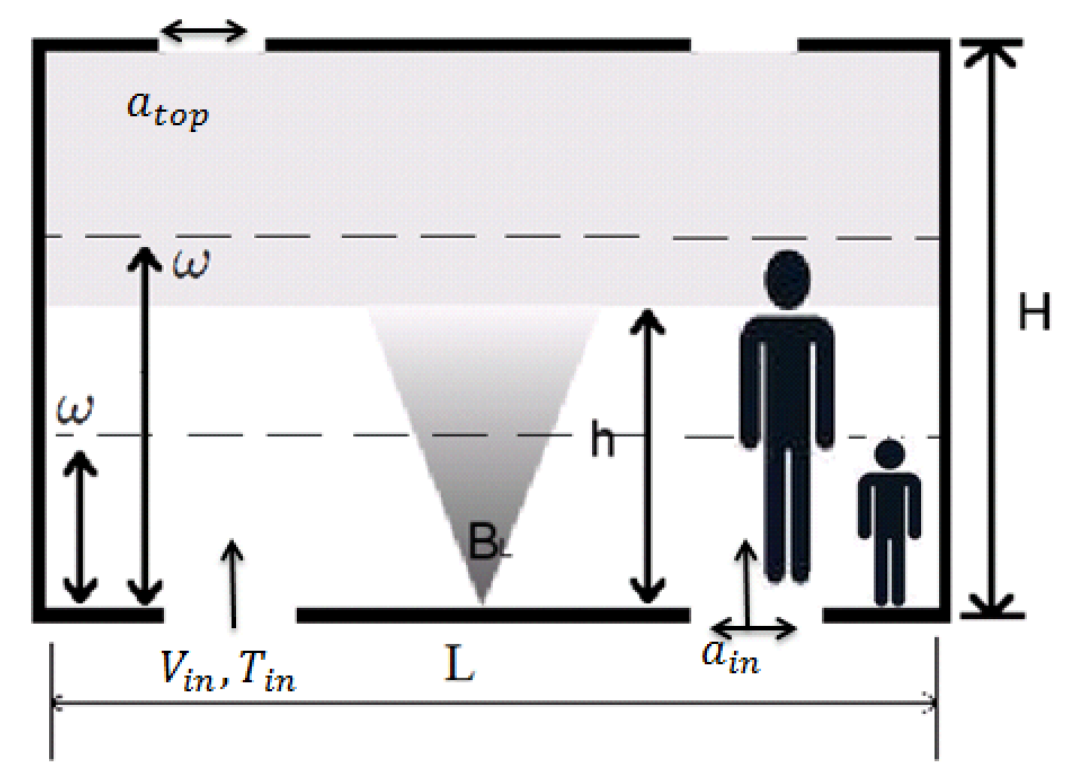

Figure 1: Schematic of displacement ventilation from a plume rising from a line source on the floor, showing the key variables for the problem domain $\mathbb{D}$. The variable $h$ is the interface height. The parameter $\omega$ is the height of the region of interest $\Omega$, which is chosen to reflect the occupant region. Here we show two possible choices of $\omega$.

$$
Q=D(B)^{1 / 3} y,
$$

where $y$ is the vertical coordinate and $D=(2 \alpha)^{\frac{2}{3}}$ is a universal constant dependent on the entrainment coefficient $\alpha$. Consistent with Linden et al. [26, we assume that a steady state is eventually reached.

By opening the doorway and turning on the heat source, two qualitatively different types of flow are generated. The plume, due to density differences, ascends toward the ceiling while the air in the ambient is entrained into the plume resulting in continuous mixing. When the plume reaches the ceiling it spreads out toward the end walls and modifies the temperature in the room. Over time and as the buoyant convection and entrainment continues there will be a descending front. In the mean time, a vertical flow is injected in the room from the inlet. During the transient evolution, therefore, there exists a vertically moving (assumed) horizontal interface below which the incoming fluid refreshes constantly until the fluid in the lower layer reaches equilibrium with the inlet fluid, and above which the fluid is lighter (warmer) than the inlet fluid. Eventually, the interface height reaches a steady value and so does the temperature of the upper layer, typically after some further time. (Here, we focus on the steady state and plan to study the transient emptying box problem in the future). At steady state and within the rising plume, (whose width is assumed to be negligible compared to the cross-sectional area of the room) the flow direction is upward. Outside the plume, the horizontal component of velocity is toward the plume, due to the entrainment process. Above the interface (and outside the plume), the vertical component of velocity is downward, while below the interface, the vertical velocity is upward, due to the momentum of the inlet flow. In each layer, i.e. above and below the interface, the vertical component of velocity vanishes as the interface approaches. We label the interface height as $h$.

Since the interface vertical velocity is zero outside the plume, at steady state the flow rate through the upper vent should match the volume flow rate in the plume at the interface. Moreover, the buoyancy flux (or heat flux) through the upper vent should balance that of the plume at the interface height. Hence, the temperature in the upper layer must have the same value everywhere, and therefore the plume should behave as a momentum jet in this region as the upper layer temperature is dictated by the plume temperature at $y=h$. Hence, in our simplified model the temperature field is given by 


$$
T=\left\{\begin{array}{ll}
T_{i n} & y \leq h \\
\left.T_{p}\right|_{y=h}=T_{i n}+k_{L} h^{-1} & y>h
\end{array},\right.
$$

where $T_{p}$ is the plume temperature, and $k_{L}=\frac{T_{r e f} B^{2 / 3}}{g D}$. Eq. 33 implies that at the lower layer the temperature matches that of the inlet, and at the upper layer it equals that of the plume at the interface height to ensure buoyancy (energy) conservation.

Equating the inlet volume flux per width $Q_{i n}=a_{i n} V_{i n}$ we find the interface height to be

$$
h=\left(\frac{Q_{i n}}{D B^{1 / 3}}\right) .
$$

In contrast to the results of Ref. 26, our model predicts that for specified inlet conditions, the interface height is a function of the source strength $B$, although it is possible to show that Eqs. (3) and (4) are consistent with the results of Ref. [26] in the limit of exchange with exterior. Finally, we define an effective area (per unit length), $a_{i n}^{*}$, as

$$
a_{i n}^{*}=\frac{a_{i n} a_{t o p}}{\sqrt{0.5\left(a_{t o p}^{2}+a_{i n}^{2}\right)}} .
$$

Although there is no explicit appearance of $a_{i n}^{*}$ in (4), nevertheless we express the results in following sections in terms of this effective area to be consistent with previous dynamically-driven interior/exterior studies. In deriving our model we assume an incompressible flow where the temperature disparities are small so that the Boussinesq approximation holds. The flow is inviscid and the diffusion in the scalar transport equation can be ignored. The ambient stratification remains stable with increasing temperature from bottom to top. The flow within the plume is assumed to be one-dimensional and in vertical direction. The horizontal flow obeys the Taylor hypothesis for the entrainment [32].

\section{Numerical Solution : Validation and Flow Regimes}

In this section, we discuss the details of numerical solution of the mixed-convection problem introduced in the last section, and provide a comparison of our numerical results with experimentally-verified analytical solutions.

\subsection{Governing equations}

The internal flow is governed by the Boussinesq equations:

$$
\begin{aligned}
\nabla . \mathbf{V} & =0, \\
(\mathbf{V} . \nabla) \mathbf{V}+\nabla p-\beta_{00} g\left(T-T_{r e f}\right) \hat{\mathbf{y}}-\nu_{e f f} \nabla^{2} \mathbf{V} & =\mathbf{0}, \\
\mathbf{V} . \nabla T-\kappa e f f \nabla^{2} T & =0,
\end{aligned}
$$

where $\mathbf{V}=\left(V_{1}, V_{2}\right)$ is the fluid velocity, $T$ is the temperature, and $\hat{\mathbf{y}}$ is the unit vector in the vertical direction. Here, the flow is characterized by the parameters $\nu_{\text {eff }}$ and $\kappa_{\text {eff }}$, the effective viscosity and diffusivity, which can accommodate eddy viscosity turbulence models. In this study, we use the Reynolds Averaged-NavierStokes (RANS) formulation to calculate $\nu_{\text {eff }}$ and $\kappa_{\text {eff }}$. We use the standard $k-\epsilon$ closure model [34] and hence, all the variables in Eq. 6 are mean (time-averaged) values.

We select the two-variable turbulence models since they have been validated for indoor environment 2, and are also computationally feasible. In this study, our focus is on statistically steady ventilation problems. 
We note that other computational frameworks, such as Large Eddy Simulation (LES) models, are also popular for built environment simulation. However, our extensive validation via comparison of numerical results with analytical plume models, and that of frozen-turbulence based RANS optimization with finitedifference sensitivities, gives us confidence that our choice of turbulence models is appropriate for the class of problems discussed here. Moreover, the impact of buoyancy on $k$ and $\epsilon$ equations is consistent with [35] where the buoyancy production is assumed to be proportional to vertical temperature gradient.

The equation set 6 is subject to the following boundary conditions

$$
\begin{array}{r}
\text { inlet }: \mathbf{V}=\mathbf{V}_{i n}, T=T_{i n},(\mathbf{n} . \nabla) p=0, \\
\text { outlet }:(\mathbf{n} . \nabla) \mathbf{V}=\mathbf{0},(\mathbf{n} . \nabla) T=0, p=0, \\
\text { wall }: \mathbf{V}=\mathbf{0},(\mathbf{n} . \nabla) T=0,(\mathbf{n} . \nabla) p=0, \\
\text { source }: \mathbf{V}=\mathbf{0},(\mathbf{n} . \nabla) T=q^{\prime \prime} / k,(\mathbf{n} . \nabla) p=0,
\end{array}
$$

where $q^{\prime \prime}$, representative of the heat source, is the heat flux per length, $k$ is the conductivity of the wall and and $\mathbf{n}$ is a unit normal vector. For select cases, we also consider a Dirichlet boundary condition for the source, i.e. $T=T_{s}$ is implemented on the source region of the wall. Finally, for $k$ and $\epsilon$ boundary conditions, we used standard wall functions with a turbulence intensity of 5 percent at inlet.

We define the following non-dimensional variables:

$$
\mathbf{V}^{*}=\frac{\mathbf{V}}{V_{r e f}}, \theta=\frac{T-T_{r e f}}{\Delta T_{r e f}}, p^{*}=\frac{p}{\rho_{0} V_{r e f}^{2}}, x *=\frac{x}{L_{r e f}},
$$

where $V_{r e f}, \Delta T_{r e f}, L_{r e f}$ are reference/characteristic velocity, temperature difference and length, respectively. We re-write the equation in non-dimensional form (dropped asterisks)

$$
\begin{aligned}
\nabla . \mathbf{V} & =\mathbf{0}, \\
(\mathbf{V} . \nabla) \mathbf{V}+\nabla p-\frac{G r}{R e^{2}} \theta \hat{\mathbf{y}}-\frac{1}{R e} \nabla^{2} \mathbf{V} & =0, \\
\mathbf{V} . \nabla \theta-\frac{1}{P e} \nabla^{2} \theta & =0,
\end{aligned}
$$

Here, the key parameters are

$$
\begin{array}{r}
R e=\frac{V_{r e f} H}{\nu}, \\
P e=R e \cdot P r=\frac{V_{r e f} H}{\kappa}, \\
R i=\frac{G r}{R e^{2}}=\frac{g \beta_{00} \Delta T_{r e f} H}{V_{r e f}^{2}},
\end{array}
$$

i.e. the Reynolds number, the Péclet number and the Richardson number respectively. Here, the Richardson number is defined as the ratio of the Grashof number to the square of the Reynolds number.

\subsection{Details of numerical solver}

We use the OpenFOAM [36] software framework, which is based on the finite-volume method [37] and offers object-oriented implementations that suits the employed continuous adjoint formulation. Pressure and velocity are coupled using the Semi-Implicit Method for Pressure-Linked Equations (SIMPLE) 38] technique in the state 'direct' equations and the adjoint equations. For the convection terms, second order Gaussian integration is used with the Sweby limiter [39] to account for the propagation of density fronts and numerical stability. For the diffusion terms, Gaussian integration with central-differencing-interpolation is used. The advective terms in the energy equation are discretized using the second order upwind scheme of van Leer 40. The discretized algebraic equations are solved using the Preconditioned bi-conjugate gradient (PBiCG) method as is used by [41. 


\subsection{Comparison of numerical and analytical results}

The two-layer stratification model derived in Section 2 is only valid for certain inlet flow and plume conditions. For large inlet volume or momentum flux we expect that forced convection due to the inlet flow becomes dominant, and consequently, the plume dynamics plays a negligible role leading to a completely well-mixed room. In contrast, for very small values of inlet volume flux, the plume dynamics is expected to be the dominant phenomenon, and a transient 'filling box' stable stratification throughout the room is expected, with an asymptotic flow field of the type suggested by Baines and Turner [25]. Therefore, for either 'strong' or 'weak' ventilation through the vents the two-layer picture does not hold. As mentioned in the introduction, our goal is to develop a numerical optimization framework for buoyancy-driven flows, and validate it with optimization of experimentally-verified analytical models. Before moving on to optimization, we first obtain the parameter regime in which both models predict a strong stable stratification and an interface between the top and bottom layer. We call this regime the 'intermediate regime' since the ventilation and plume flows both affect the steady state. For this purpose, we use two non-dimensional parameters: the velocity ratio, $V_{r}$ and the scaled interface height $\zeta$, defined as

$$
V_{r}=\frac{V_{\text {in }}}{V_{p, \max }}, \quad \zeta=\frac{h}{H},
$$

where $V_{p, \max }$ is the maximum of the velocity determined along the vertical line along the centerline of the plume. It should be noted that $\zeta$ is also equivalent to a volume flux ratio $\zeta=\frac{Q_{i n}}{\max \left(Q_{p}\right)}=\frac{Q_{i n}}{D B^{1 / 3} H}$, by using Eq. 4. The reason for the choice of $V_{r}$ and $\zeta$ to characterize the intermediate regime is based on our hypothesis that the momentum and volume flux of the inlet, scaled by that of the plume, are key factors for the existence of two-layer steady state solution. As we shall see, there is a set of $V_{r}$ and $\zeta$ values, as representatives of momentum and volume flux, respectively, for which the model given in Section 2 is valid.

A symmetry plane is introduced at the midplane to save computational cost. Therefore, we only show the left half of the domain in the results of this section. The height of the interface is measured at the far left-hand side of the box (away from the plume and the incoming air), and is defined as the height at which there is minimal vertical motion, i.e. $\left.V_{2}\right|_{h} \approx 0$. We first vary $a_{i n}^{*}$ to change $\zeta$ while keeping $V_{r}$ constant. The interface height as a function of $a_{i n}^{*}$ is plotted in Fig. $2 \mathrm{a}$ and the streamlines colored according to scaled temperature are shown in Fig. $3 \mathrm{a}$-d for different values of $a_{i n}^{*}$. From Fig. 2a, it can be seen that the interface height varies approximately linearly with respect to $a_{i n}^{*}$ (or $Q_{i n}$ ); this numerical result is consistent with Eq. 4 in the analytical model. For smaller or larger values of $a_{i n}^{*}$, the non-dimensional interface height, respectively, approaches 0.09 or 0.6 based on our findings, which are the limit of the intermediate regime and in the following we explore the physical meaning behind such ratios. Therefore deviation from the linear trend is expected in such cases. There is a small offset (approximately $0.06 \mathrm{H}$ ) which may be interpreted as a 'virtual origin' for the plume consistently with previous studies, (see for example Kaye and Hunt 42 . for further discussion). For $\zeta \geq 0.6$ the interface height is not clearly identified. The temperature and velocity profile for this case is shown in Fig. 3 $\mathrm{d}$. It is seen that by increasing the inlet area, the plume becomes increasingly detached from the upper layer ambient fluid, and at a critical value of the interface height (Fig. 3d), the horizontal velocity towards the plume becomes negligible. This suggests that the plume theory foundations, e.g. entrainment hypothesis, self-similarity of velocity profiles, etc. do not hold for this parameter value, and the plume is now completely isolated from the room fluid, which manifests itself as bypassing of the inlet flow away from the plume and flow directly towards the outlet. This phenomenon is seen to exist for all values of source buoyancy flux, and only depends on $\zeta$. Hence, $\zeta \approx 0.6$ is the upper limit for the intermediate regime.

Next, we obtain the range of $V_{r}$ values corresponding to the intermediate regime. For a given buoyancy flux, we first calculate $V_{p, \max }$. Then, we vary $V_{r}$ by altering both $a_{i n}$ and $V_{i n}$ while keeping their product $Q_{i n}=a_{i n} V_{i n}$ constant. In Fig. $2 \mathrm{p}$, we plot the maximum velocity within the plume as a function of $B$ for two values of inlet velocity, $V_{i n}$. The interface height can be identified for all values of source buoyancy flux considered in Fig. $2 \mathrm{~b}$, and the results fall within the intermediate regime. As expected from plume theory [32], $V_{p, \max }$ varies with the power law of $1 / 3$ in both cases, although one case has a two times higher inlet 


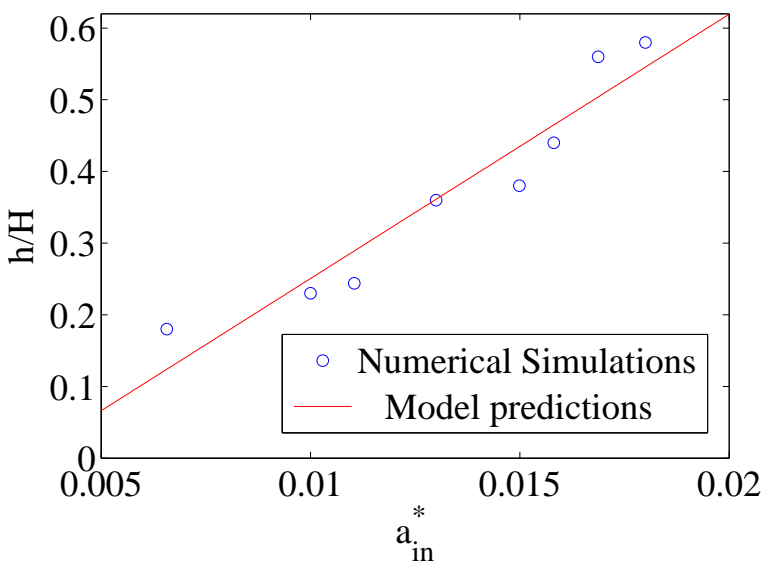

(a)

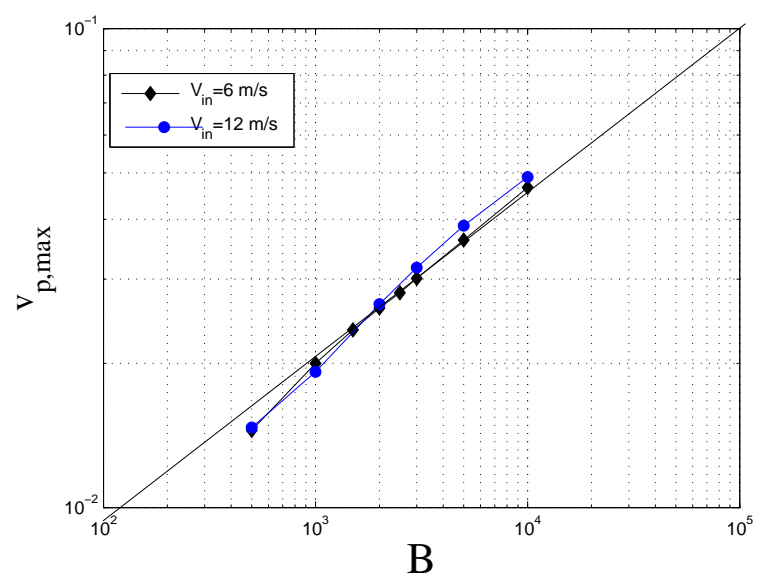

(b)

Figure 2: a) Interface height, $\zeta$, as a function of inlet effective area, $a_{i n}^{*}$. b) Maximum velocity in the plume, $V_{p, \max }$ as a function of source buoyancy flux $B$ plotted using log-log axes. Also shown is a line with a slope of $1 / 3$.

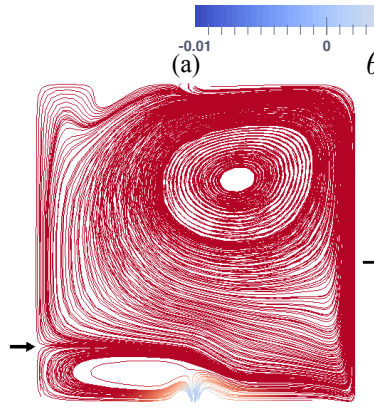

(c)

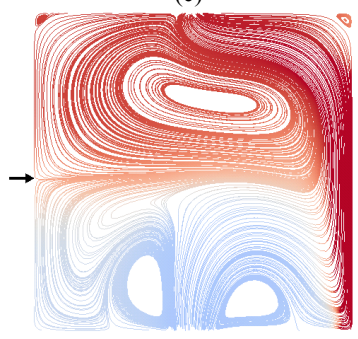

(b) ${ }^{0.02}$

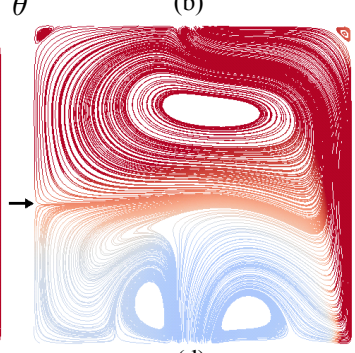

(d)

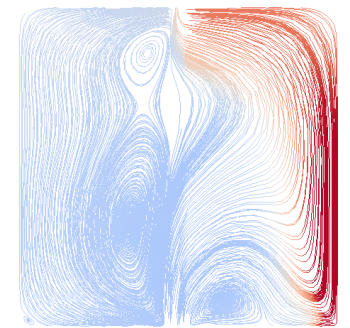

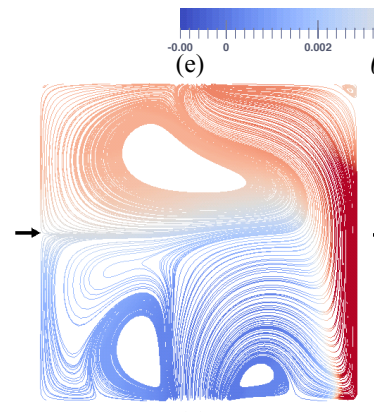

(g)

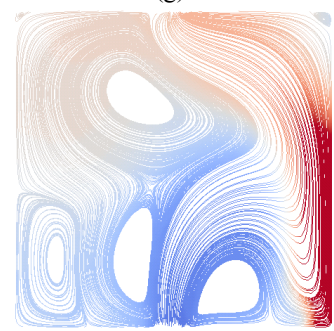

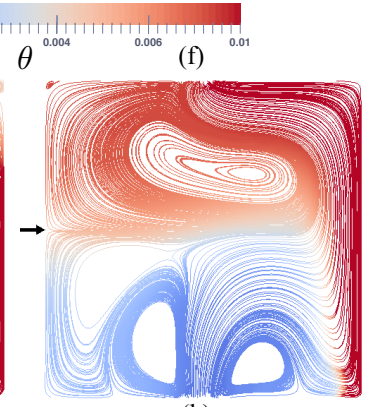

(h)

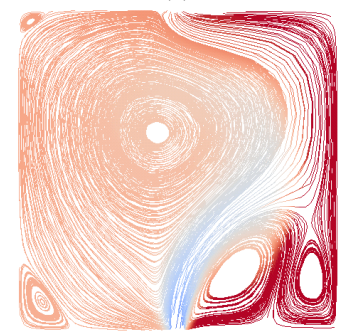

Figure 3: Streamlines colored by scaled temperature for various values of effective area $a_{i n}^{*}$ (a-d) and $V_{r}$ (e-h). a) $a_{i n}^{*}=0.0066$ and $\zeta=0.12$, b) $a_{i n}^{*}=0.0158$ and $\zeta=0.5$, c) $a_{i n}^{*}=0.0163$ and $\zeta=0.6$, d) $a_{i n}^{*}=0.0174$ and $\zeta$ is not defined. e) $V_{r}=0.72$ and $\zeta=0.52, \mathrm{f}) V_{r}=1.07$ and $\left.\zeta=0.52, \mathrm{~g}\right) V_{r}=1.44$ and $\zeta$ is not defined, and h) $V_{r}=3.85$ and $\zeta$ is not defined. The interface height is indicated by an arrow in each figure.

volume flux. This is further confirmation that for the intermediate regime, our numerical models agree with classical plume theory. Numerical results are shown in Fig. 3 e-h for the cases where $\zeta$ is kept constant and $V_{r}$ is altered. It can be seen that beyond a certain value of $V_{r}$, the interface is no longer apparent, and hence, corresponding parameters are not in the intermediate regime. When the vertical velocity of the ambient fluid at the interface becomes comparable to the maximum velocity of the plume, the vertical velocity may not be downward in the lower layer. The fountain dynamics undergoes a transition and becomes a jet at the inlet. This is consistent with Ref. [43] who observed the same phenomenon, i.e. the transition of a fountain to a jet based on the momentum flux of the injection (although they used a somewhat different Froude number 
criterion to characterize the transition).

To summarize the above discussion, for relatively large $\zeta$, characterized by the ratio of the inlet volume flux to that of the plume at interface, the flow leaves the intermediate regime due to the breakdown of the entrainment hypothesis. Also, for large values of $V_{r}$, representative of the ratio of the inlet momentum flux to the maximum momentum flux of the plume, the inflow acts as a jet instead of a fountain and the two-layer stratification is disrupted. We show a phase diagram to illustrate the domain of validity of the intermediate regime in Fig. 4. The middle area, i.e. moderate values of $\zeta$ and $V_{r}$, defines the intermediate regime, while for the region that falls outside of the marked area no well-defined interface can be seen in our numerical simulations. Finally, for small values of volume or momentum flux when $\zeta<0.09, V_{r}<0.1$, our numerical results show that the impact of the incoming flow is negligible and no clear displacement ventilation is evident.

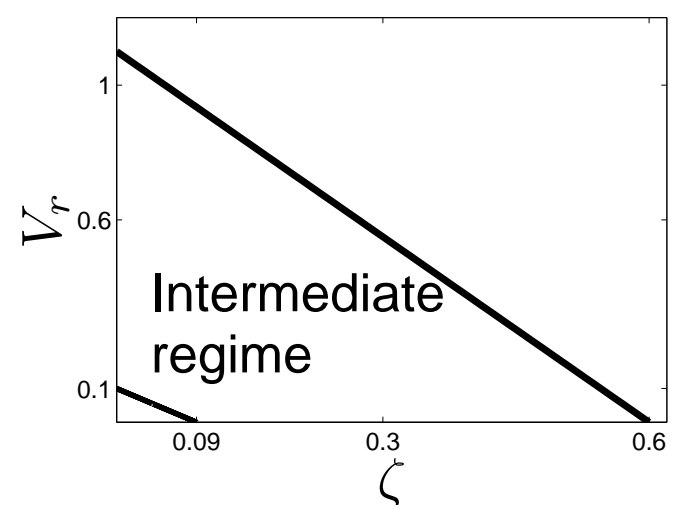

Figure 4: Phase diagram for $\zeta$, representative of the inlet volume flux and $V_{r}$, representative of the momentum flux at the inlet, showing the existence of an intermediate regime and two-layer stratification. The analytical model holds only in the intermediate regime.

\section{Optimization Problem Formulation: Direct-Adjoint-Looping and Analytical Approaches}

We describe an optimization problem that is subsequently solved via both analytical and numerical approaches, enabling a comparison with experimentally validated analytical models. The region of interest, denoted by $\Omega$, is a rectangular region that spans the length of the room, and reaches up to a specified height $\omega$. The parameters $T_{d}$ and $\mathbf{V}_{d}$ denote the desired temperature and velocity respectively, to be maintained in $\Omega$.

We define the cost function as

$$
J=\alpha^{\prime} \int_{\Omega}\left\|\mathbf{V}-\mathbf{V}_{d}\right\|^{2} d x d y+\gamma \int_{\Omega}\left(T-T_{d}\right)^{2} d x d y
$$

where \|\| is the Euclidean L2 norm. For consistent dimensions $\gamma=\frac{V_{r e f}^{2}}{\Delta T_{r e f}^{2}} \beta^{\prime}$, where $\alpha^{\prime}$ and $\beta^{\prime}$ are nondimensional weight. The optimization problem is then stated as

$$
\begin{array}{r}
\text { minimize } \mathcal{J}=\mathcal{J}(\mathcal{W}, \mathcal{U}) \\
\text { s.t. } \mathcal{R}(\mathcal{W}, \mathcal{U})=0
\end{array}
$$


where $\mathcal{W}=(\mathbf{V}(x, y), p(x, y), T(x, y))$ are the state variables, and $\mathcal{U}$ is the set of design variables, i.e., $\mathcal{U}=\left(V_{i n}, T_{i n}\right) . \quad$ It should be remembered that the horizontal component of $V_{i n}$ is zero and hence the inlet velocity is unidirectional, and so it is appropriate to express this velocity as a scalar. $\mathcal{R}$ denotes the constraints imposed by the governing equations, corresponding to Boussinesq Eq. (6) in the case of the numerical optimization, and the plume Eqs. (3-4) in the case of analytical optimization. Additional constraints may also be implemented with no change in the formulation of Eq. (13). We thus have a constrained optimization problem.

The predicted mean vote (PMV) is commonly used as a measure of thermal comfort in the literature 44. Such models consider air velocity and temperature as parameters affecting thermal comfort, as well as other parameters, e.g. relative humidity. Here, however, as a proxy for thermal comfort, we measure the discomfort in the room as the deviation of $T$ from $T_{\text {comf }}$, which is defined as the comfortable temperature. We ignore the velocity component in the objective function of thermal comfort, but later we add a constraint on the product of temperature and velocity, as a placeholder for an energy constraint.

\subsection{Direct-Adjoint-Looping (DAL) Formulation}

We tackle the optimization problem in Eq. $(12)$ by use of the Lagrangian $\mathcal{L}$. We reformulate the optimization problem as

$$
\operatorname{minimize} \mathcal{L}=\mathcal{J}+\left\langle\mathcal{P}^{\top}, \mathcal{R}\right\rangle
$$

where $\mathcal{P}=\left(\mathbf{V}_{a}, p_{a}, T_{a}\right)$ is the vector of adjoint variables, and we use the notation $\langle f, g\rangle=\int_{\mathbb{D}} f g d x d y$. It should be noted that we only identify the boundary conditions that (locally) minimize $\mathcal{J}$ and there is no closed-loop control in the current study. The adjoint variables are Lagrange multipliers to enforce the state Eq. (6). To ensure the (at least local) optimality of the solution, we enforce $\delta \mathcal{L}=\delta_{\mathcal{U}} \mathcal{L}+\delta_{\mathcal{W}} \mathcal{L}=0$, where $\delta G$ denotes variation of a dependent variable $G$. We choose the adjoint variables such that $\delta_{\mathcal{W}} \mathcal{L}=0$. The sensitivity equations with respect to design variables are then obtained as $\delta \mathcal{L}=\delta_{\mathcal{U}} \mathcal{L}$. This procedure can be generalized to any number of design variables, and hence contributes to a significant saving in computational effort when the number of design variables is large. This idea is at the heart of the adjoint method [15, 16, 17] (refer to appendix A for details of our derivation).

By requiring that first order variations with respect to the state variables vanish at optimal solutions, i.e., $\delta_{\mathcal{W}} \mathcal{L}=0$, we obtain

$$
\begin{array}{r}
\nabla . \mathbf{V}_{a}=0 \\
-(\mathbf{V} . \nabla) \mathbf{V}_{a}+\nabla \mathbf{V}^{\top} \cdot \mathbf{V}_{a}+\nabla p_{a}+T \nabla T_{a}+\nu_{e f f} \nabla^{2} \mathbf{V}_{a}+\alpha^{\prime}\left(\mathbf{V}-\mathbf{V}_{d}\right)=0 \text { on } \Omega \\
-\nabla \mathbf{V}^{\top} \cdot \mathbf{V}_{a}+(\mathbf{V} . \nabla) \mathbf{V}_{a}+\nabla p_{a}+T \nabla T_{a}+\nu_{e f f} \nabla^{2} \mathbf{V}_{a}=0 \text { on } \mathbb{D} \backslash \Omega \\
\mathbf{V} . \nabla T_{a}+\kappa_{e f f} \nabla^{2} T-g \beta_{00}\left(T-T_{r e f}\right)+\gamma\left(T-T_{d}\right)=0 \text { on } \Omega \\
\mathbf{V} . \nabla T_{a}+\kappa_{e f f} \nabla^{2} T-g \beta_{00}\left(T-T_{r e f}\right)=0 \text { on } \mathbb{D} \backslash \Omega .
\end{array}
$$

The adjoint boundary conditions are

$$
\begin{array}{r}
\text { inlet : } \mathbf{V}_{a}=0, T_{a}=0,(\mathbf{n} . \nabla) p_{a}=0 \\
\text { outlet }: V_{n} V_{a, t}+\nu_{e f f}(\mathbf{n} . \nabla) V_{a, t}=0, \\
T_{a} V_{n}+\kappa_{e f f}(\mathbf{n} . \nabla) T_{a}=0, \\
p_{a}=V_{n} V_{a, n}+V \cdot V_{a}+\nu_{e f f}(\mathbf{n} . \nabla) V_{a}, \\
\text { wall : } \mathbf{V}_{a}=0,(n . \nabla) T_{a}=0,(\mathbf{n} . \nabla) p_{a}=0,
\end{array}
$$

where the subscripts $n, t$ denote normal and tangential components, respectively. 
Now we define non-dimensional variables,

$$
\mathbf{V}_{a}^{*}=\frac{\mathbf{V}_{a}}{L_{r e f}}, T_{a}^{*}=\frac{T_{a} \nabla^{2} T_{r e f}}{V_{r e f} H}, p_{a}^{*}=\frac{p_{a}}{V_{r e f} L_{r e f}}, x *=\frac{x}{L_{r e f}} .
$$

and the resulting non-dimensional adjoint equations are (dropping asterisks as before):

$$
\begin{array}{r}
\nabla \cdot \mathbf{V}_{a}=0 \\
-(\mathbf{V} . \nabla) \mathbf{V}_{a}+\nabla \mathbf{V}^{T} \cdot \mathbf{V}_{a}+\nabla p_{a}+T_{a} \nabla \theta-\frac{1}{R e} \nabla^{2} \mathbf{V}_{a}+2 \alpha^{\prime}\left(\mathbf{V}-\mathbf{V}_{d}\right)=0 \text { on } \Omega, \\
-(\mathbf{V} . \nabla) \mathbf{V}_{a}+\nabla \mathbf{V}^{T} \cdot \mathbf{V}_{a}+\nabla p_{a}+T_{a} \nabla \theta-\frac{1}{R e} \nabla^{2} \mathbf{V}_{a}=0 \text { on } \mathbb{D} \backslash \Omega, \\
R i \mathbf{V}_{a}-\mathbf{V} \cdot \nabla T_{a}-\frac{1}{P e} \nabla^{2} T_{a}+2 \beta^{\prime}\left(\theta-\theta_{d}\right)=0 \text { on } \Omega, \\
R i \mathbf{V}_{a} \cdot \hat{\mathbf{y}}-\mathbf{V} \cdot \nabla T_{a}-\frac{1}{P e} \nabla^{2} T_{a}=0 \text { on } \mathbb{D} \backslash \Omega
\end{array}
$$

Here we have made the choice of using the 'frozen turbulence' hypothesis [45]. Hence, we require that $(k(x, y), \epsilon(x, y))$, which are field variables solved along with $(\mathbf{V}(x, y), p(x, y), T(x, y))$ during the forward or direct simulation, have the same values during the solution of the adjoint equation in the DAL framework. When the number of design variables is small, an assessment of the validity of this assumption can be done for a given problem by comparing adjoint sensitivities to those computed using a finite-difference method. We relegate this comparison to appendix B. We note that a more comprehensive analysis of the validity of the frozen turbulence hypothesis is needed for problems with a higher number of design variables or more complicated, possibly time-dependent optimization problems. To validate this hypothesis in a systematic fashion would entail adding adjoint equations for $(k, \epsilon)$ to the set of adjoint equations. A first step in this direction was taken in Ref. [46], where a toy-model for a RANS-type closure scheme was considered. In that study, certain energy-exchange mechanisms between the mean-flow and turbulent eddies were found to be present only in the adjoint optimization performed without the frozen-turbulence hypothesis.

In the momentum Eq. (18), diffusion is scaled with the inverse of the Reynolds number, or the inverse of the Péclet number for the temperature equation. Hence, by increasing the Reynolds number, the adjoint advection operator, i.e. $-(\mathbf{V} . \nabla) \mathbf{V}_{a}+\nabla \mathbf{V}^{T} \cdot \mathbf{V}_{a}$ dominates in the velocity equations, and $-\mathbf{V} . \nabla T_{a}$ dominates in the temperature equation. Consequently, in numerical implementations, to prevent numerical instabilities, care should be taken in using the correct mesh size, particularly for flows with higher Re.

After computation of the adjoint field we obtain the sensitivity of the cost function with respect to inlet velocity and temperature, i.e. the design variables, as follows

$$
\nabla_{V_{i n}} \mathcal{J}=\left.p_{a}\right|_{\text {in }}-\left.\frac{1}{R e}(\mathbf{n} . \nabla) V_{a}\right|_{\text {in }}, \quad \nabla_{T_{i n}} \mathcal{J}=\left.\frac{1}{P e}(\mathbf{n} . \nabla) T_{a}\right|_{\text {in }}
$$

We illustrate the iterative solution procedure schematically in Fig. 5 . Determination of a solution begins with an initial guess for the design variables $\left(V_{i n}, T_{i n}\right)$. The set of 'direct' state and adjoint equations are solved in a loop and the subsequent sensitivity calculation is used to obtain the next guess for the optimal design variables. This process is repeated until the convergence criterion for the cost functional is satisfied, i.e. $\left|\mathcal{J}^{n+1}-\mathcal{J}^{n}\right| \leq \epsilon$.

\subsection{Analytical optimization for the reduced model}

The analytical model derived in Section 2 is now employed to find the optimal inlet conditions, i.e. $T_{i n}^{o}$ and $V_{i n}^{o}$. We focus on a local comfort problem, and set $\alpha^{\prime}=0$ in Eq. $(12)$, with $T_{d}=T_{\text {comf }}$. The objective function for thermal comfort problems is then written as

$$
J=\int_{\Omega}\left(T-T_{\text {comf }}\right)^{2} d x d y .
$$




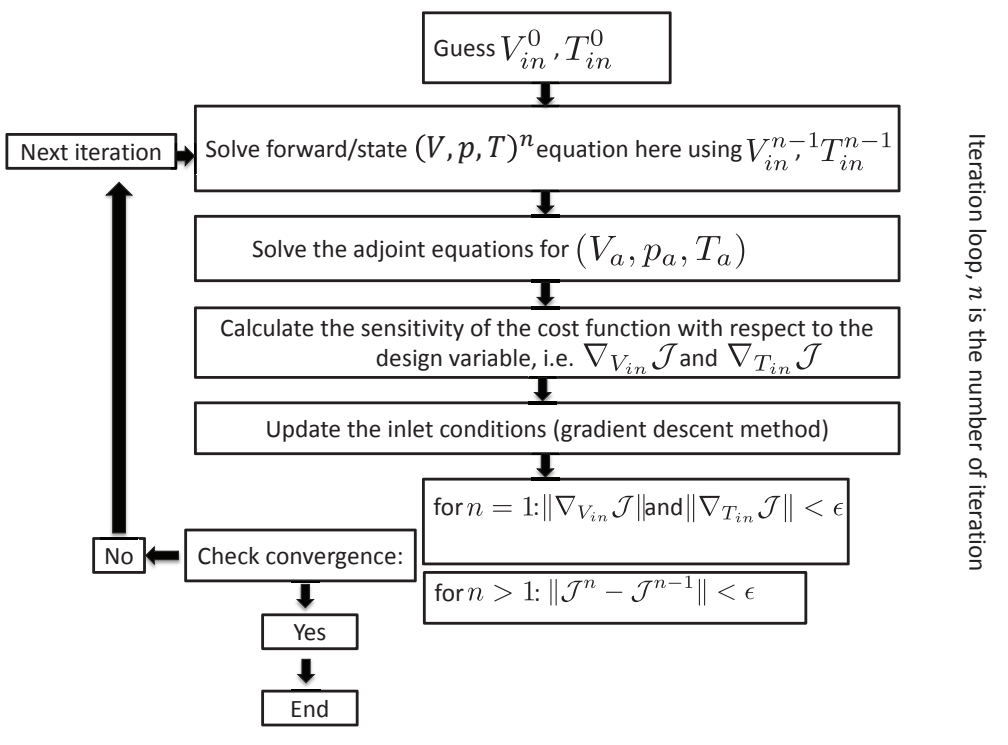

Figure 5: Flow chart for the Direct-Adjoint-Looping method.

In the following discussion, we consider two cases: optimization without any constraint on the set of possible inlet conditions, and then optimization with such a constraint imposed. The specific constraint we consider is on the incoming energy of the flow, expressed in terms of enthalpy flux. That is, for the constrained problem, we require

$$
T_{i n} V_{i n}=d,
$$

where $d$ is a given constant value proportional to enthalpy flux.

\subsubsection{Unconstrained optimization problem}

Recall that the temperature distribution is given by Eq. (3), which is a discontinuous distribution, with $T=T_{i n}$ in the lower layer, $z<h$. We rewrite Eq. 44 as $h=a V_{i n}$ with $a=\frac{a_{\text {in }}}{D B^{1 / 3}}$. It is clear that the minimum value of the cost function Eq. 20$)(=0)$ is achieved for $T=T_{\text {comf }}$, and ensuring that $h \geq \omega$. Since there are no constraints on the inlet variables, simply setting $T_{\text {in }}=T_{\text {comf }}$, and $V_{\text {in }} \geq \omega / a$ leads to the optimal thermal comfort solution.

\subsubsection{Constrained optimization problem}

We consider the enthalpy-constrained version of the optimization problem with a cost function given by Eq. 20, and the constraint given in Eq. 21. We begin by recalling the equations characterizing optimal solutions in a nonlinear constrained optimization problem with equality and inequality constraints. Consider the following problem:

$$
\text { minimize } f(x) \text {, s.t. } \psi_{j}(x) \leq 0 \text { for } j=1, \ldots, m \text { and } \phi_{i}(x)=0 \text { for } i=1, \ldots, l \text {. }
$$

The Lagrangian is defined as

$$
\mathcal{L}=f(x)+\lambda^{\top} \psi+\mu^{\top} \phi
$$


Then $x^{o}$ is a local minimum if and only if there exists a unique $\lambda^{o}$ s.t.

$$
\begin{aligned}
\nabla_{x} \mathcal{L}\left(x^{o}, \mu^{o}, \lambda^{o}\right) & =0, \\
\lambda^{o} & \geq 0, \\
\lambda^{o \top} \psi & =0, \\
\psi\left(x^{o}\right) & \leq 0, \\
\phi\left(x^{o}\right) & =0 .
\end{aligned}
$$

These five equations characterize a local optimal solution according to Karush-Kuhn-Tucker (KKT) conditions 47.

Since the temperature distribution given by Eq. (3) is discontinuous, the optimization problem is solved in two stages. We first assume $\psi=a V_{i n}-\omega \leq 0$, i.e. the optimal inlet velocity is such that the resulting interface height is less than or equal to the height of the region of interest. The Lagrangian $\mathcal{L}$ for this case is

$$
\mathcal{L}=\mathcal{J}+\lambda\left(a V_{i n}-\omega\right)+\mu\left(T_{i n} V_{i n}-d\right) .
$$

The last term is added due to the enthalpy flux constraint, and $\mu$ is the corresponding Lagrange multiplier. Substituting the temperature distribution given in Eq. (3) into Eqs. [25], we obtain

$$
\mathcal{L}=\left(T_{\text {in }}-T_{\text {comf }}\right)^{2} \omega+2\left(T_{\text {in }}-T_{\text {comf }}\right)\left(\frac{k_{L}}{a V_{i n}}\right)\left(\omega-a V_{i n}\right)+\left(\frac{k_{L}}{a V_{i n}}\right)^{2}\left(\omega-a V_{i n}\right)+\lambda\left(a V_{i n}-\omega\right)+\mu\left(T_{i n} V_{i n}-d\right) .
$$

In the second stage, we apply the KKT condition Eq. $24\left(\right.$ a)) i.e. $\nabla_{T_{i n}, V_{i n}} \mathcal{L}=0$, to obtain

$$
\begin{aligned}
& \frac{\partial \mathcal{L}}{\partial T_{i n}}=2\left(T_{i n}-T_{\text {comf }}\right) \omega+2\left(\frac{k_{L}}{a V_{i n}}\right)\left(\omega-a V_{i n}\right)+\mu V_{i n}=0, \\
& \frac{\partial \mathcal{L}}{\partial V_{i n}}=\frac{-2 k_{L}^{2} \omega}{a^{2} V_{i n}^{3}}-\frac{k_{L}}{a V_{i n}^{2}}\left(2 \omega\left(T_{i n}-T_{\text {comf }}\right)-k_{L}\right)+a \lambda+\mu T_{i n}=0 .
\end{aligned}
$$

It can be shown that Eq. 27 results in $\frac{\partial \mathcal{L}}{\partial V_{i n}}=\frac{-k_{L}^{2}}{a V_{i n}^{2}}+a \lambda+\mu\left(T_{i n}+\frac{k_{L}}{a V_{i n}}\right)=0$. From the KKT condition Eq. 24(c)), we obtain either

$$
\lambda^{o}=0, \psi\left(x^{o}\right) \leq 0, \text { or } \lambda^{o}>0, \psi\left(x^{o}\right)=0 .
$$

where $x^{o}=\left\{T_{i n}, V_{i n}\right\}$. If $\lambda^{o}=0$, we obtain

$$
\begin{array}{r}
V_{i n}^{o}=\frac{\omega\left(\frac{k_{L}}{a}+d\right)}{-\frac{k_{L}^{2}}{2\left(k_{L}+a d\right)}+k_{L}+T_{\text {comf }} \omega}, \\
T_{i n}^{o}=d \frac{-\frac{k_{L}^{2}}{2\left(k_{L}+a d\right)}+k_{L}+T_{\text {comf }} \omega}{\omega\left(\frac{k_{L}}{a}+d\right)} .
\end{array}
$$

Conversely, if $\psi\left(x^{o}\right)=0$, we obtain

$$
V_{\text {in }}^{o}=\frac{\omega}{a}, T_{i n}^{o}=\frac{a d}{\omega}, \lambda^{o}=\frac{k_{L}^{2}}{\omega^{2}}+2 \frac{\left(a d+k_{L}\right)}{\omega\left(\frac{a d}{\omega}-T_{\text {comf }}\right)}>0 .
$$

For both these situations, it is still necessary to check that the assumption $a V_{i n}^{o} \leq \omega$ holds. 
We next consider the case when $\psi=\omega-a V_{i n} \leq 0$. For this case, the Lagrangian is

$$
\mathcal{L}=\int_{0}^{\omega}\left(T_{i n}-T_{\text {comf }}\right)^{2} d y+\lambda\left(\omega-a V_{\text {in }}\right)+\mu\left(T_{i n} V_{\text {in }}-d\right) .
$$

Once again applying the KKT condition Eq. $\left(24\right.$ (a)), i.e. $\nabla_{T_{i n}, V_{i n}} \mathcal{L}=0$, we obtain

$$
\frac{\partial \mathcal{L}}{\partial T_{\text {in }}}=2\left(T_{\text {in }}-T_{\text {comf }}\right) \omega+\mu V_{\text {in }}=0, \frac{\partial \mathcal{L}}{\partial V_{\text {in }}}=-a \lambda+\mu T_{\text {in }}=0 .
$$

Similarly to before, if $\psi\left(x^{o}\right)=0$, we obtain

$$
V_{i n}^{o}=\frac{\omega}{a}, T_{i n}^{o}=\frac{d a}{\omega}, \mu^{o}=-2 a\left(T_{i n}^{o}-T_{\text {comf }}\right) \lambda^{o}=-2 T_{i n}^{o}\left(T_{i n}^{o}-T_{\text {comf }}\right)>0,
$$

where the last equation holds when $T_{\text {comf }}>\frac{d a}{\omega}$. If $\lambda^{o}=0$, we obtain

$$
T_{\text {in }}^{o}=T_{\text {comf }}, V_{\text {in }}^{o}=\frac{d}{T_{\text {comf }}} .
$$

Depending upon the values of $\Pi_{1}=\frac{d a}{\omega T_{\text {comf }}}$ and $\Pi_{2}=\frac{a\left(\frac{k_{L}}{a}+d\right)}{-\frac{k_{L}^{2}}{2\left(k_{L}+a d\right)}+k_{L}+T_{c o m f} \omega}=\frac{a V_{i n}^{o}}{\omega}$, the enthalpyconstrained optimization has one of the three following solutions.

- Case $1: \Pi_{1} \geq 1$, which yields $T_{i n}^{o}=T_{\text {comf }}$.

- Case 2: $\Pi_{1}<1$ and $\Pi_{2} \leq 1$, giving $T_{i n}^{o}=d\left[\frac{-\frac{k_{L}^{2}}{2\left(k_{L}+a d\right)}+k_{L}+T_{\text {comf }} \omega}{\omega\left(\frac{k_{L}}{a}+d\right)}\right]$. This occurs for smaller values of $k_{L}$; in the limit $k_{L} \rightarrow 0, T_{\text {in }}^{o}=T_{\text {comf }}$.

- Case 3: $\Pi_{1}<1$ and $\Pi_{2}>1$, giving $T_{i n}^{o}=\frac{d a}{\omega}$. This occurs for larger values of $k_{L}$.

In all of the above cases, the value of the optimal inlet velocity $V_{i n}^{o}$ is obtained by using the enthalpy flux constraint.

\section{Results and Discussion}

\subsection{Inverse Design}

As a first step towards validating the DAL method optimization framework, we use the method to solve an inverse-design problem. We choose arbitrary inlet conditions $\left(T_{i n}^{o}, V_{i n}^{o}\right)$ and solve the forward problem Eq. 66 to obtain $\left(V^{d}, T^{d}\right)$. We employ the DAL method to optimize the cost function given by Eq. 12. Since the optimal solution is known to be $\left(T_{i n}^{o}, V_{i n}^{o}\right)$, this procedure serves as a method to validate the numerical optimization framework.

For this test case, we use $V_{i n}^{o}=12 \mathrm{~m} / \mathrm{s}$ (vertical velocity only) and $T_{i n}^{o}=300 \mathrm{~K}$ as the inlet conditions. The region of interest height is $40 \%$ of that of the room. We start the DAL method from a guess which is reasonably different compared to the optimal solution, i.e. $T_{i n}=305 \mathrm{~K}$. Fig. 6 shows that after a few iterations, the optimal inlet conditions are automatically recovered. 


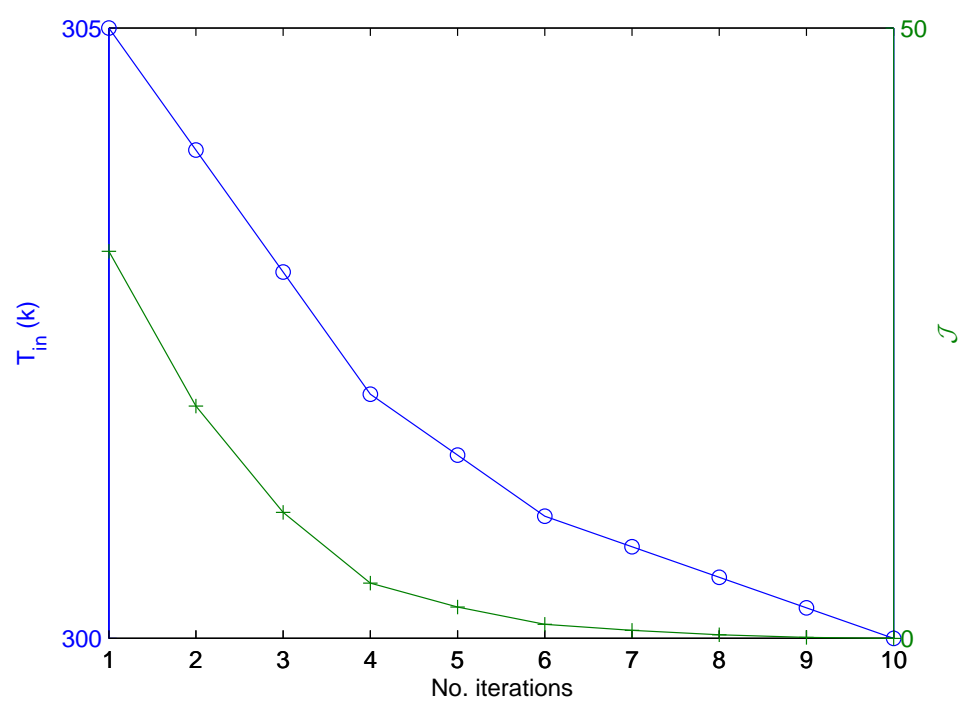

Figure 6: Variation of $T_{i n}$ (blue circles) and cost function $\mathcal{J}$ (green crosses) with iteration loop number of the DAL method iteration.

\subsection{Optimal Design for Local Thermal Comfort}

For unconstrained optimization, the numerical DAL method results in $T_{\text {in }}^{o}=T_{\text {comf }}$. This is consistent with the analytical model results presented in Section 4.2.1, with one difference. The sensitivity of the cost function with respect to the inlet velocity, i.e., $\left.\frac{\partial \mathcal{L}}{\partial V}\right|_{i n}$ is always positive. Hence, the unconstrained optimization will lead to a state where the whole room is at $T_{\text {comf }}$, a situation which is clearly neither in the intermediate regime, nor of practical interest.

We now discuss the extension of the DAL method to the more meaningful constrained optimization problem discussed in Section 4.2.2. The Lagrangian is written as (also see Appendix A)

$$
\mathcal{L}=\mathcal{J}+<\mathcal{P}^{\top}, \mathcal{R}>+\lambda\left(T_{\text {in }} V_{\text {in }}-d\right)
$$

The sensitivity with respect to inlet velocity is

$$
\left.\frac{\partial \mathcal{L}}{\partial V}\right|_{\text {in }} \delta \mathbf{V}_{i n}=\left.\frac{\partial \mathcal{J}}{\partial V}\right|_{\text {in }} \delta \mathbf{V}_{i n}+<P^{\top}, \frac{\partial R}{\partial \mathcal{W}}>\left.\right|_{\text {in }} \delta \mathcal{W}+\lambda T_{i n} \delta \mathbf{V}_{i n}
$$

which yields

$$
\left.\frac{\partial \mathcal{L}}{\partial V}\right|_{\text {in }}=-p_{a} \mathbf{n}+\left.\nu_{e f f} \frac{\partial \mathbf{V}_{a}}{\partial n}\right|_{\text {in }}+\lambda T_{i n} \mathbf{n}
$$

Similarly

$$
\left.\frac{\partial \mathcal{L}}{\partial T}\right|_{\text {in }}=-\left.\kappa_{e f f} \frac{\partial T_{a}}{\partial n}\right|_{\text {in }}+\lambda V_{i n}
$$

From Eqs. 36 and (37), we obtain

$$
\lambda=-\frac{-p_{a}+\nu_{e f f} \frac{\partial V_{a, n}}{\partial n}}{T_{i n}}
$$


and

$$
\left.\frac{\partial \mathcal{L}}{\partial T}\right|_{\text {in }}=-\left.\frac{\nu_{e f f}}{p r_{t}} \frac{\partial T_{a}}{\partial n}\right|_{\text {in }}+\frac{d}{T_{i n}^{2}}\left(-p_{a}+\nu_{\text {eff }} \frac{\partial V_{a, n}}{\partial n}\right) .
$$

Eq. (39) computes the true gradient with respect to the inlet temperature, and it is used to improve the next guess in the constrained DAL method. Note that the corresponding guess for inlet velocity is obtained from the enthalpy flux constraint.

We consider several cases for the local comfort problem. The simulation time for a typical case, consisting of 30800 finite volumes, is $14336.9 \mathrm{sec}$ on a compute node with 10 CPUs each with the clock speed of $3.458 \mathrm{GHz}$ for one iteration. Several iterations may be required for DAL to converge depending on the initial guess. These cases are presented in Table 1 . It should be noted that any arbitrary region may be chosen as $\Omega$. However, to enable comparisons with the analytical model, we restrict the choice of $\Omega$ to be a rectangular region spanning the length of the room, and height $\omega$. For all cases we define $\theta_{i n}=$ $\left(T_{\text {in }}-T_{\text {comf }}\right) /\left(T_{s}-T_{\text {comf }}\right)$.

\begin{tabular}{c|c|c|c|c|c|c|c}
\hline case & $d$ & $\zeta$ & $\omega$ & $\theta_{\text {in }}^{o}$ & $\mathcal{J}^{o}$ & $T_{s}$ & $T_{\text {comf }}$ \\
\hline case 1 & 3600 & 0.372 & 0.4 & -0.01 & $4.65 \mathrm{e}-6$ & 305 & uniform \\
case 2 & 3600 & 0.372 & 0.8 & -0.02 & $7.32 \mathrm{e}-6$ & 305 & uniform \\
case 3 & 3600 & 0.372 & 0.2 & 0 & $4.14 \mathrm{e}-06$ & 305 & uniform \\
case 4 & 1000 & 0.168 & 0.4 & -0.08 & $1.29 \mathrm{e}-5$ & 305 & uniform \\
case 5 & 1000 & 0.168 & 0.8 & -0.1 & $1.85 \mathrm{e}-5$ & 305 & uniform \\
case 6 & 3600 & 0.304 & 0.4 & -0.02 & $1.87 \mathrm{e}-5$ & 310 & uniform \\
case 7 & 3600 & 0.244 & 0.4 & -0.1 & $8.13 \mathrm{e}-5$ & 320 & uniform \\
case 8 & 4500 & 0.360 & 0.4 & -0.02 & $1.53 \mathrm{e}-5$ & 310 & uniform \\
case 9 & 3600 & 0.360 & 0.4 & -0.20 & $5.65 \mathrm{e}-5$ & 305 & linear \\
case 10 & 3600 & 0.360 & 0.4 & -0.16 & $1.62 \mathrm{e}-5$ & 305 & parabolic \\
\hline
\end{tabular}

Table 1: Summary of optimization results for the local thermal comfort problem. $d$ is the constraint on the inlet conditions imposed by Eq. 21].

For case 1 , the initial guess was taken to be $\theta_{i n}=10$, which is far from the actual optimal solution. This case allows us to study the behavior of the DAL method in a large neighborhood of the optimal solution to investigate the efficiency of the method when the initial guess is chosen not close to $\theta_{i n}^{o}$. As shown in Fig. 7. after some iterations, $\mathcal{J}$ becomes very small such that there is negligible decrease in the cost function for further iterations. This is also evident in panel (b) of Fig. 8 where the minimum of $\mathcal{J}$ in panel (a) corresponds to $\nabla \mathcal{J}=0$ in panel (b). For the sake of comparison, we also start from another initial guess $\theta_{i n}=-0.5$. Similarly, the minimum is achieved with the same value of $\theta_{i n}^{o}=0.01$. In Fig. 9 the streamlines corresponding to the optimal values of case 1,2 and 3 are depicted. For smaller values of $\omega,\left|\theta_{i n}^{o}\right|$ is almost zero implying $T_{i n}^{o} \approx T_{\text {comf }}$. By increasing $\omega,\left|\theta_{i n}^{o}\right|$ increases. This is because for a fixed amount of enthalpy flux, the interface height cannot always be raised up to $\omega$ while maintaining $T_{i n}=T_{\text {comf }}$. In such a case, a fraction of the upper layer also resides in the region of interest, and hence $T_{i n}^{o}$ is different than $T_{\text {comf }}$.

We now consider the impact of enthalpy flux, $d$, on the optimal solutions. As shown in Fig. 9, when comparing case 1 and 4, it is evident that for lower $d$, the interface height is below the region of interest. An extreme case is case 5 , where $\theta_{i n}^{o}=-0.1$. In this case, the optimal inlet temperature is significantly lower than $T_{\text {comf }}$. Moreover, for larger values of $d, J^{o}$ is lower, as also indicated in Table 1 Comparison of case 8 and case 6 , both at the same $T_{s}$ but different $d$, reveals that for larger values of $d, \mathcal{J}$ decreases and the interface height increases, as was also seen for $T_{s}=305$. 


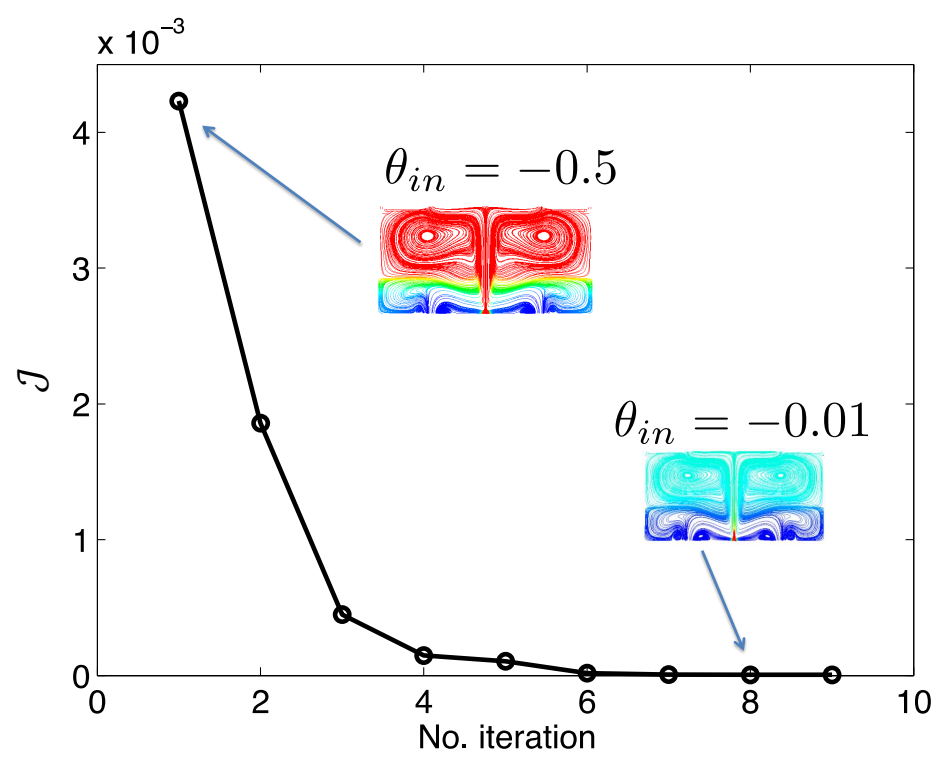

Figure 7: Variation of the cost function $\mathcal{J}$ with the iteration number of the DAL method. The arrows indicate the corresponding streamlines colored by $\theta$ for two select values of $\theta_{i n}$. The colorbar is the same as that of Fig. 9a-h.

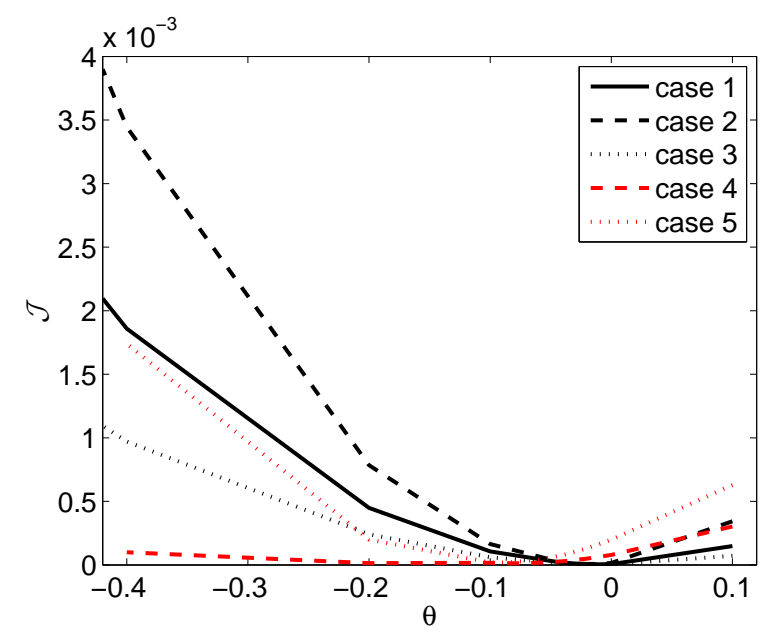

(a)

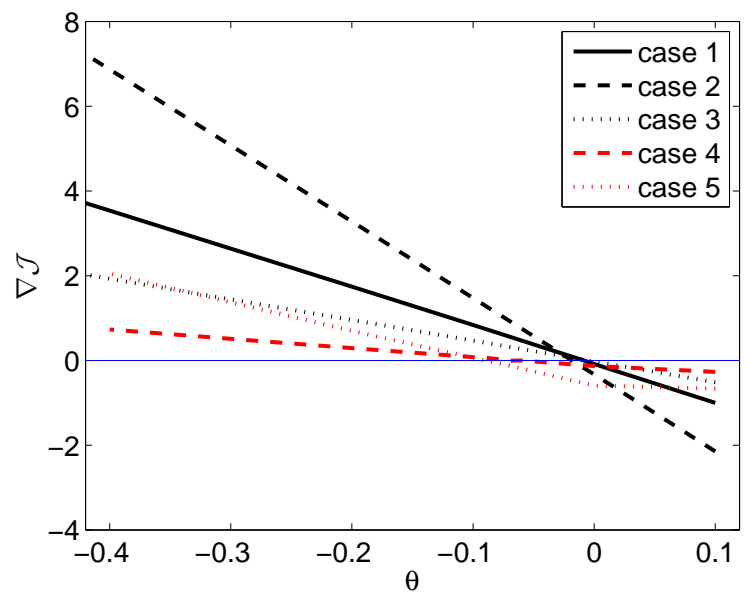

(b)

Figure 8: Variation with $\theta$ of: a) cost function $\mathcal{J}$; and b) $\nabla \mathcal{J}$, for several different cases. The blue horizontal line in panel (b) indicates $\nabla \mathcal{J}=0$.

Next, we consider the impact of source temperature $T_{s}$ on optimal solutions. In cases 6 and 7 , we increase the value of $T_{s}$, while keeping the rest of the parameters the same as in case 1 . The heat load is larger, which leads to the requirement of the injection of colder air into the room, resulting in a larger $\left|\theta_{i n}^{o}\right|$ and larger $\mathcal{J}$, by comparison with the situation in case 1 . We also see that increases in buoyancy source strength lead to a lower interface height, which is consistent with the analytical model, i.e. Eq. (4). In Fig. 10, we provide a comparison between optimal inlet temperatures for the bounded optimization problems determined using the DAL method and the optimal inlet temperatures determined using analytical optimization. On average, the relative error is less than 10 percent. We define relative error as $\frac{\left|\theta_{i n}^{o}\right|_{D A L}-\left.\theta_{i n}^{o}\right|_{\text {model }} \mid}{\theta_{i n}^{o} \mid \text { model }}$ with $\left.\theta_{i n}^{o}\right|_{D A L}$ and $\left.\theta_{i n}^{o}\right|_{\text {model }}$ as optimal inlet values based on DAL and analytical model, respectively. The relative error 


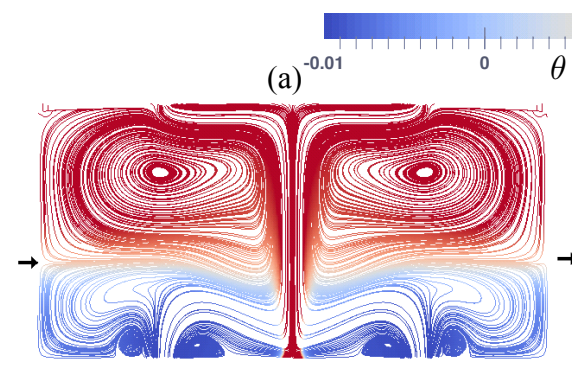

(c)

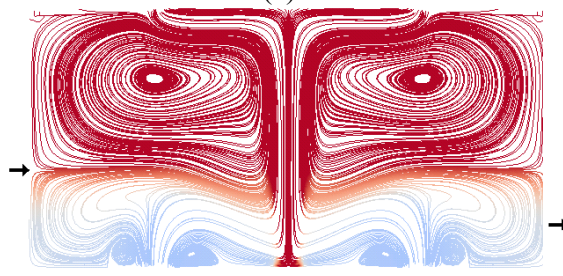

(e)

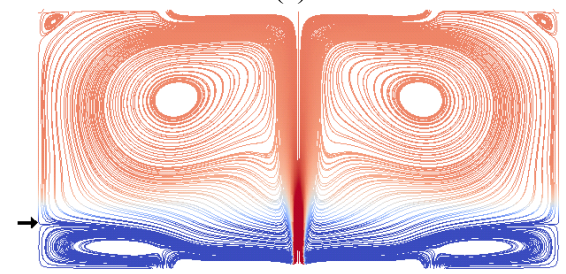

(g)

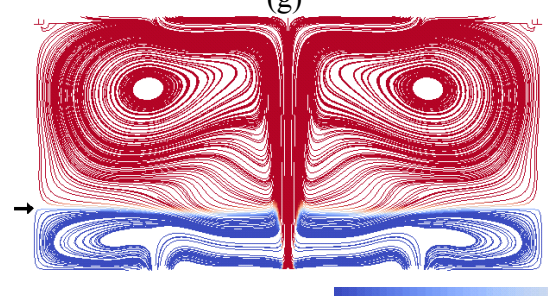

(i) -0.20

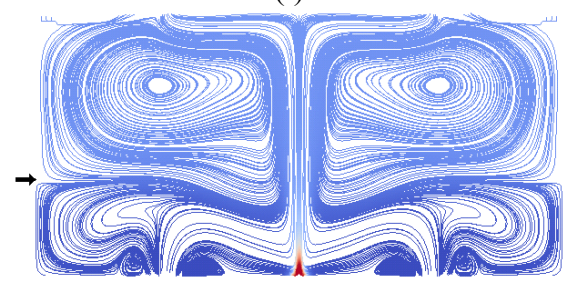

(b)

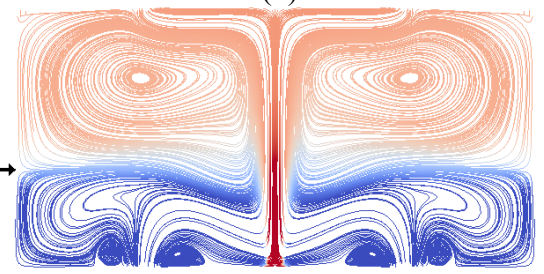

(d)

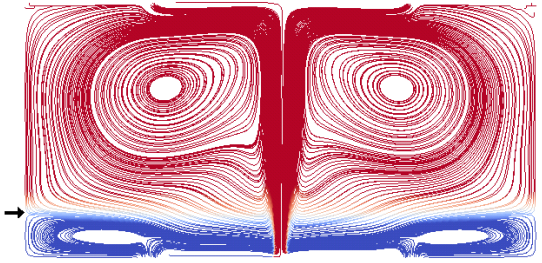

(f)

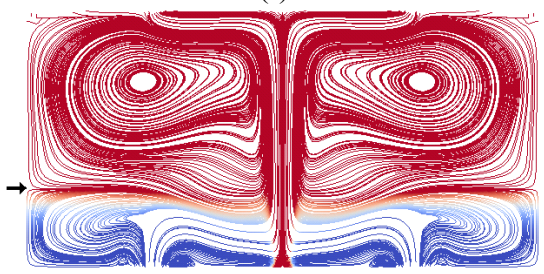

(h)

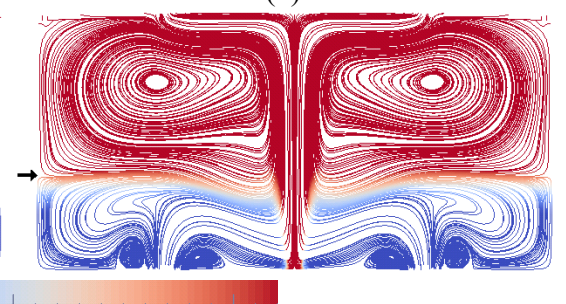

${ }^{0.1} \theta \quad{ }^{0} \quad{ }^{0.02}(\mathrm{j})$

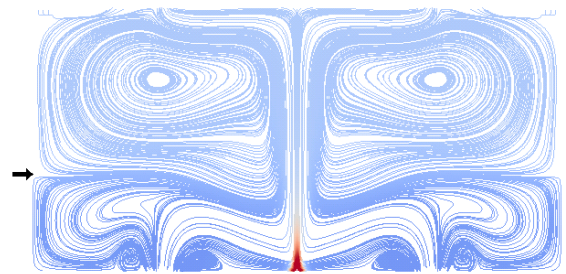

Figure 9: Streamlines (colored by $\theta$ ) of the optimal answer determined by the DAL method for: a) case 1; b) case 2; c) case 3 ; d) case 4 ; e) case 5 ; f) case 6 ; g) case 7 ; h) case 8 ; i) case 9 ; and j) case 10 . The arrow indicates the elevation of interface height. The color bar for panels a-h is at the top and for panels $\mathrm{i}$ and $\mathrm{j}$ is at the bottom.

increases with increasing values of $\omega$. The reason for this behaviour of the relative error is that the analytical model naturally predicts two layers which are assumed to be well-mixed. Therefore, for the analytical model, the impact of the source is manifested as the temperature jump across the interface. Conversely, for the numerical solution, there is no discontinuity in the temperature profile. Hence, for larger depths of the region of interest, the error associated with temperature calculation increases. The source strength does not alter the error significantly, proving that for the intermediate regime, the plume model employed in deriving the analytical model is accurate, consistent with a long line of other studies [26, 24, 27].

Heretofore, we have assumed the desired temperature is a constant field with $T=T_{\text {comf }}$ everywhere 


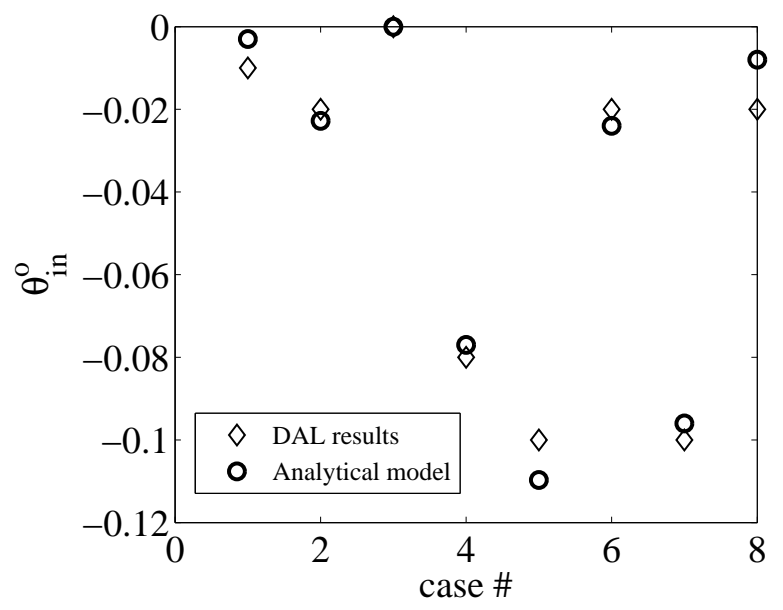

Figure 10: Comparison of optimal inlet temperature, $\theta_{i n}^{o}$, for various cases listed in Table 1

in the region of interest $\Omega$. According to ASHRAE standard 55 [48, Thermal Environmental Conditions for Human Occupancy, air temperature differences between the head level $(1.1 \mathrm{~m}$ for seated and $1.7 \mathrm{~m}$ for standing occupants) and ankle level $(0.1 \mathrm{~m})$ should not exceed 3 degrees. Accordingly, we consider two $T_{\text {comf }}$ profiles which have 3 degrees temperature difference between floor and ceiling:

$$
\begin{aligned}
& T_{\text {comf }}(z)=298.5+6 y, \\
& T_{\text {comf }}(z)=299+12 y^{2} .
\end{aligned}
$$

The two temperature profiles are taken to be linear and parabolic functions of height, having the same average temperature. In Fig. 9 cases 9 and 10 corresponding to these linear and parabolic profiles are also shown, and the behaviour of the system in each case is qualitatively similar to the systems with constant desired temperature in the region of interest.

\section{Conclusions and Future Work}

In this paper, we have studied the problem of optimizing the temperature and velocity distribution in a domain containing turbulent buoyancy-driven flow associated with a plume rising from a line heat source. As examples of the utility of the DAL method, we compute 'optimal' velocities and temperatures of the inlet flow, constrained to be at fixed enthalpy value, which lead to minimization of a cost function that penalizes deviation from a desired temperature and velocity field in part of the domain, the 'region of interest'.

To validate our numerical methodology, we develop a reduced-order analytical model for the system. This analytical model is based on the assumption of strong stratification, and predicts an interface separating a well-mixed lower layer from a well-mixed upper layer. We demonstrate that there exists a range of parameter values for which the numerical and analytical models agree, which we refer to as the "intermediate regime". A phase diagram based on two non-dimensional parameters representing relative inlet volume and momentum flux is then constructed. The intermediate regime is shown to exist for small to moderate values of these two parameters. It is found that for large values of the inlet volume flux, the plume becomes isolated from the room fluid, and for large values of momentum flux, the fountain dynamics breaks down and the inflow acts as a pure jet instead. Analytical solutions for the optimal inlet velocity and temperature can then be derived in this intermediate regime using the analytical model. The choice of RANS turbulence model is a problem-specific task. For displacement ventilation flows standard $k-\epsilon$ or RNG $k-\epsilon$ models have been used, see for example [49. In the intermediate regime, and in terms of results shown in Fig. 2, we do not observe a significant model-dependence, but one should be cautious to always include a valid and 
appropriate turbulence model. The DAL method can be extended to any RANS two-variable turbulence modes in a straightforward manner, should one need to consider a different approach.

An initial validation of the numerical optimization method is done using an inverse-design problem. We show that the DAL method recovers the design inlet velocity and temperature exactly. Several cases of enthalpy-constrained optimization are then studied. If the enthalpy is chosen to be high enough, the optimal temperature is seen to be approaching the desired temperature in the region of interest, and the interface height is at or above the upper boundary of that region. For a fixed value of inlet enthalpy, increasing the height of the region of interest results in an optimal solution where part of the upper layer is inside that region. We also compute optimal solutions for cases where the desired temperature increases linearly or quadratically with height. In the intermediate regime, it is found that the optimal solutions from numerical and analytical optimization are within $10 \%$ of each other.

The test case we consider is the displacement ventilation, due to its vast practical importance [24]. In displacement ventilation, the direct supply of ambient cold air and resulting temperature stratification in the building may be associated with thermal discomfort [50]. For the case where inlet energy/enthalpy is not constrained, the displacement ventilation may break down, and the stratified two layer room may turn into mixing ventilation [51. Therefore, we focus on the practically relevant constrained optimization of displacement ventilation. For a given heat load in the room, provided that $H$ is large enough so that the set of parameters are in the intermediate regime, our algorithm optimizes temperature distribution in the occupied region under the constraint of fixed enthalpy flux of the inlet.

Implementing a correct thermal comfort model requires consideration of several parameters such as air temperature, velocity, relative humidity, clothing, and even mindset of occupants, which in turn necessitates consideration of additional constraints that is beyond the scope of the present manuscript and demands its own specific research. Of course, various efforts have been made in this direction [52]. However, the conclusion to be drawn from Laftchiev and Nikovski discussion [52] is that different techniques yield different degrees of thermal satisfaction. Our proposed framework is able to accommodate more complicated cost functions as equations are derived in terms of variations with respect to velocity and temperature of a general, unspecified cost function $\mathcal{J}$, i.e. $\frac{\partial \mathcal{J}}{\partial V}, \frac{\partial \mathcal{J}}{\partial \theta}$, in Appendix A. An important feature of the current modeling is the objective function can be expressed in terms of field variables and hence, not only more complicated thermal comfort and energy consumption, but also air quality problems can be tackled with a similar approach.

For an initial guess that is far from the optimal solution, numerical challenges may be observed. Our numerical results show that if the deviation of the initial guess from the optimal solution is significant, a very refined mesh is required for convergence of the adjoint equations. We believe that the reason is the source terms in Eq. 18 become much larger than other terms, which necessitates a very accurate mesh. However, within a reasonable vicinity of optimal solution, and as is evident from the analytical optimization results, there is no multiplicity. Our knowledge about the physics of the problem considered in this study assists us to start with a guess that does not result in numerical problems.

This work is a first step towards the development of a validated numerical optimization and control framework for turbulent indoor airflow dynamics. The close agreement of the optimal solutions computed using the DAL method for this test-case with those obtained from experimentally validated analytical models provides motivation for further development of numerical optimization tools for optimal design of the indoor environment. Nevertheless, several challenging problems need to be solved before the methods described in this work can be applied in realistic settings. For example, there exist several analytical models for transient ventilation dynamics, and the various dynamical behaviors are well-understood. The numerical optimization of transient problems has much higher computational burden than the steady state cases considered here, and are natural candidates for numerical reduced-order modeling, especially in the context of real-time control. Furthermore, we have employed the frozen turbulence assumption in our numerical optimization method. While we confirm the validity of this approach via comparison with finite-difference sensitivity computations, more work needs to be done in order to identify conditions under which this approach breaks down. It is plausible that some optimal solutions to certain classes of indoor airflow optimization problems, e.g. those at least related to optimal mixing, may be found only by using the full set of adjoint equations, including those corresponding to the turbulence variables such as $(k, \epsilon)$. Of course, this issue is not just confined to 
indoor airflow. A vast array of practical engineering problems involve turbulent flow, and optimization using direct numerical simulation models is not feasible in most cases. The extension of our framework to deal with these issues will be a topic of future work.

\section{A Derivation of adjoint equations}

A popular method to derive optimality conditions is the Lagrangian method [15, 17, 53. In this approach, a Lagrangian is introduced to PDE-constrained optimization to account for governing equations and other bounds on design variables. The augmented objective functional, i.e. Lagrangian, is

$$
\delta \mathcal{L}=\delta \mathcal{J}+<\mathcal{P}^{\top}, \frac{\partial R}{\partial \mathcal{W}} \delta \mathcal{W}>+\int\left(\lambda \frac{\partial \mathcal{F}}{\partial \mathcal{U}} \delta \mathcal{U}\right) \cdot \mathbf{n} d S
$$

with $\langle$. $>$ denoting volume integration and $\mathcal{F}$ is any constraint, e.g. the energy of inlet flow, other than the governing equations themselves. Here, $\hat{\mathbf{y}}$ is the unit vector in the direction of gravitational acceleration, $\mathcal{P}^{\top}=\left(V_{a}, p_{a}, T_{a}\right), \mathcal{U}^{\top}=\left(V_{i n}, \theta_{i n}\right)$ and $\frac{\partial R}{\partial \mathcal{W}}$ and $\frac{\partial \mathcal{F}}{\partial \mathcal{U}}$ are Jacobians of the constraints (the Navier-Stokes equations, the continuity equation and the energy equation) and the objective or cost functional, respectively, with respect to direct variables and input (control) variables; i.e.

$$
\frac{\partial R}{\partial \mathcal{W}} \delta \mathcal{W}=\left(\begin{array}{c}
(\delta \mathbf{V} \cdot \nabla) \mathbf{V}+(\mathbf{V} \cdot \nabla) \delta \mathbf{V}-\frac{1}{R e} \nabla^{2} \delta \mathbf{V}-(R a \hat{\mathbf{y}}) \delta \theta+\nabla \delta p \\
\nabla \cdot \delta \mathbf{V} \\
\delta \mathbf{V} \cdot \nabla \theta+\mathbf{V} \cdot \nabla \delta \theta-\frac{1}{P e} \nabla^{2} \delta \theta
\end{array}\right)
$$

Using 42,41 can be rewritten as

$$
\begin{aligned}
\delta \mathcal{L}=\delta_{\mathbf{V}} \mathcal{J}+\delta_{p} \mathcal{J} & +\delta_{\theta} \mathcal{J}+<\mathbf{V}_{a},(\delta \mathbf{V} . \nabla) \mathbf{V}+(\mathbf{V} . \nabla) \delta \mathbf{V}-\frac{1}{R e} \nabla^{2} \delta \mathbf{V}-(R a \hat{\mathbf{y}}) \delta \theta+\nabla \delta p>+ \\
& <p_{a}, \nabla . \delta \mathbf{V}>+<T_{a}, \delta \mathbf{V} \cdot \nabla \theta+\mathbf{V} . \nabla \delta \theta-\frac{1}{P e} \nabla^{2} \delta \theta>+\int\left(\lambda \frac{\partial \mathcal{F}}{\partial \mathcal{U}} \delta \mathcal{U}\right) . \mathbf{n} d S
\end{aligned}
$$

For unbounded optimization, $\mathcal{F}=0$ and hence $\frac{\partial \mathcal{F}}{\partial \mathcal{U}} \delta \mathcal{U}=0$. For the optimization constrained by an enthalpy condition, $\mathcal{F}=V_{i n} \theta_{\text {in }}$, and so

$$
\frac{\partial \mathcal{F}}{\partial \mathcal{U}} \delta \mathcal{U}=\left(\begin{array}{c}
V_{i n} \delta \theta_{i n} \\
\theta_{i n} \delta V_{i n}
\end{array}\right)
$$

Each term of 42 can be calculated analytically. Specifically, using vector calculus and integration by parts, appropriate Euler-Lagrange equations can be derived.

$$
\begin{array}{r}
\delta \mathcal{L}=<\delta \mathbf{V}, \frac{\partial \mathcal{J}}{\partial \mathbf{V}}-\nabla p_{a}-(\mathbf{V} . \nabla) \mathbf{V}_{a}+(\nabla \mathbf{V})^{\top} \mathbf{V}_{a}-\frac{1}{R e} \nabla^{2} \mathbf{V}_{a}+T_{a} \nabla \theta>+ \\
<\delta p, \nabla \cdot \mathbf{V}_{a}>+<\delta \theta, \frac{\partial \mathcal{J}}{\partial \theta}-\mathbf{V} \cdot \nabla T_{a}-\frac{1}{P e} \nabla^{2} T_{a}+(R i \hat{\mathbf{y}}) \mathbf{V}_{a}>+ \\
\int(\delta \mathbf{V})\left(p_{a}+V_{a}(\mathbf{V} . \mathbf{n})+\frac{1}{R e} \nabla \mathbf{V}_{a}\right) . \mathbf{n} d S+\int(\delta \theta)\left(T_{a}(\mathbf{V} . \mathbf{n})+\frac{1}{R e} \nabla T_{a}\right) d S
\end{array}
$$

To ensure local optimality it is required that $\delta \mathcal{L}=0$. From the space integral, the adjoint equations are recovered as 


$$
\begin{aligned}
\nabla \cdot \mathbf{V}_{a} & =0 \\
-(\mathbf{V} \cdot \nabla) \mathbf{V}_{a}+\nabla \mathbf{V}^{T} \cdot \mathbf{V}_{a}+\nabla p_{a}+T_{a} \nabla \theta-\frac{1}{R e} \nabla^{2} \mathbf{V}_{a}+\frac{\partial \mathcal{J}}{\partial V} & =0 \\
R i \mathbf{V}_{a} \cdot \hat{\mathbf{y}}-V \cdot \nabla T_{a}-\frac{1}{P e} \nabla^{2} T_{a}+\frac{\partial \mathcal{J}}{\partial \theta} & =0 .
\end{aligned}
$$

From surface integrations and after some simplifications [15, we obtain the adjoint boundary conditions:

$$
\begin{array}{r}
\text { inlet : } \mathbf{V}_{a}=0, T_{a}=0,(\mathbf{n} \cdot \nabla) p_{a}=0, \\
\text { outlet }: V_{n} V_{a, t}+\frac{1}{R e}(\mathbf{n} \cdot \nabla) V_{a, t}=0, \\
T_{a} V_{n}+\frac{1}{P e}(\mathbf{n} \cdot \nabla) T_{a}=0, \\
p_{a}=V_{n} V_{a, n}+\frac{1}{R e}(\mathbf{n} \cdot \nabla) V_{a},
\end{array}
$$

wall : $\mathbf{V}_{a}=0,(\mathbf{n} . \nabla) T_{a}=0,(\mathbf{n} . \nabla) p_{a}=0$.

\section{B Validation of the frozen-turbulence hypothesis for computing adjoint-based sensitivities}

We compare the adjoint-based sensitivities, that have been computed using the frozen-turbulence hypothesis, with those computed by a finite-difference (FD) method. Referring to the general first-order forward Euler approximation of a derivative, we obtain

$$
\begin{array}{r}
\left.\nabla_{V_{i n}} \mathcal{J}\right|_{F D}=\frac{\mathcal{J}\left(\mathbf{V}_{i n}+\delta \mathbf{V}_{i n}\right)-\mathcal{J}\left(\mathbf{V}_{i n}-\delta \mathbf{V}_{i n}\right)}{2 \delta \mathbf{V}_{\text {in }}}, \\
\left.\nabla_{T_{i n}} \mathcal{J}\right|_{F D}=\frac{\mathcal{J}\left(T_{i n}+\delta T_{i n}\right)-\mathcal{J}\left(T_{i n}-\delta T_{i n}\right)}{2 \delta T_{i n}}, \\
\left.\nabla_{V_{i n}} \mathcal{J}\right|_{a d}=\int_{\text {in }}\left(-p_{a}+\nu_{\text {eff }}(\mathbf{n} . \nabla) \mathbf{V}_{a}\right) d A \\
\left.\nabla_{V_{i n}} \mathcal{J}\right|_{a d}=\int_{\text {in }}\left(\kappa_{\text {eff }}(\mathbf{n} . \nabla) T_{a}\right) d A
\end{array}
$$

Fig. 11 shows the results of just such a comparison. Overall, the two methods give similar values for sensitivities. This confirms that our numerical framework is robust, and the assumption of frozen-turbulence in our DAL method does not introduce errors in the computation of optimization problem.

The process of choosing an appropriate step size for the finite difference method is non-trivial. There are two types of errors in the finite difference approximation: truncation error and condition error. The truncation error is the error introduced due to neglecting higher order terms in the Taylor expansion. The condition error is associated with numerical noise, and is caused by loss of numerical precision. It may be a result of computer round-off error, or the operation of subtracting large numbers which are very nearly equal. On reducing step-size, the truncation error decreases while the condition error generally increases. Therefore an estimation of each error is crucial in finite-difference evaluation of sensitivities; there can be even an optimal step size that can decrease the time and cost of the calculations. The truncation error is

$$
\begin{array}{r}
T(h)=\frac{h}{2} f^{\prime \prime(x)}, \\
f^{\prime \prime}(x) \approx \Phi=\frac{f(x+h)-2 f(x)+f(x-h)}{h^{2}},
\end{array}
$$




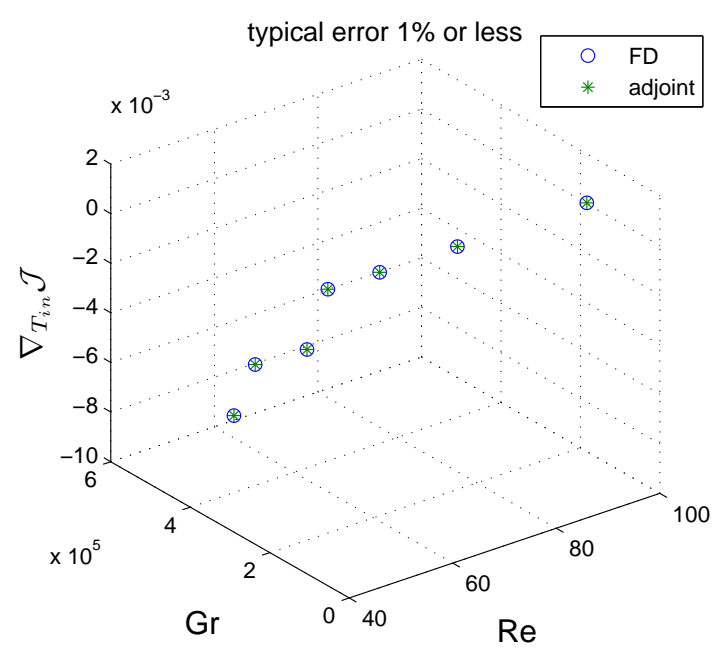

(a)

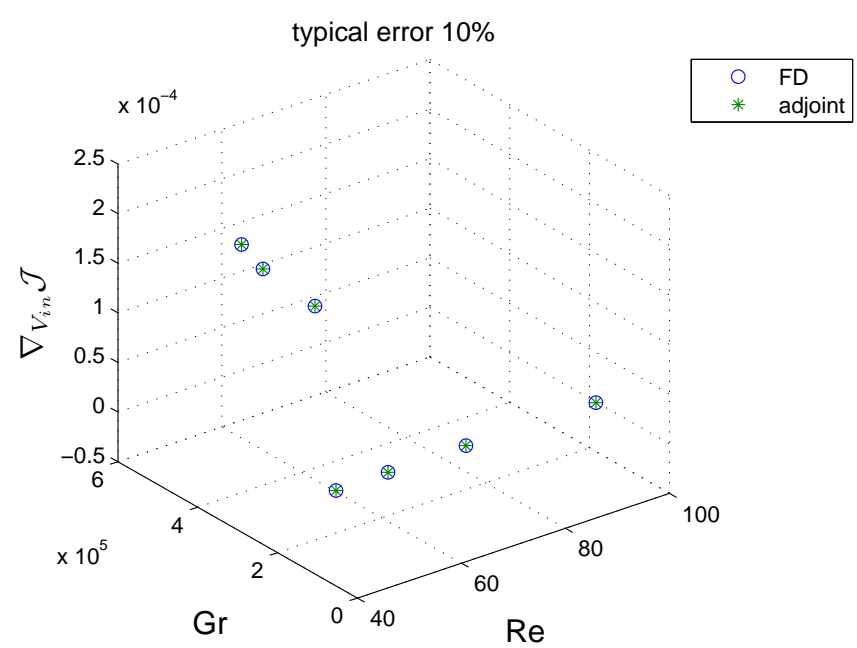

(b)

Figure 11: Comparison of adjoint-based and finite-difference-based sensitivities for validation of the adjoint method. The relative error is defined as $\epsilon=\frac{\left|\nabla_{T_{i n}} \mathcal{J}\right|_{F D}-\left.\nabla_{T_{i n}} \mathcal{J}\right|_{a d} \mid}{\left.\nabla_{T_{i n}} \mathcal{J}\right|_{F D}} \times 100$ for temperature and $\epsilon=\frac{\left|\nabla_{V_{i n}} \mathcal{J}\right|_{F D}-\left.\nabla_{V_{i n}} \mathcal{J}\right|_{\text {ad }} \mid}{\left.\nabla_{V_{i n}} \mathcal{J}\right|_{F D}} \times 100$ for velocity. Gradients with respect to inlet a) temperature, $\nabla_{T_{i n}} \mathcal{J}$, and b)velocity $\nabla_{V_{i n}} \mathcal{J}$, are shown.

while the condition error is 54

$$
C(h)=\frac{2}{h} \epsilon_{A},
$$

where $\epsilon_{A}$ is a bound on the absolute error in the computed function. Hence the total error is

$$
\begin{gathered}
e=\frac{h}{2} \Phi+\frac{2}{h} \epsilon_{A}, \\
h_{\text {opt }}=2 \sqrt{\frac{\epsilon_{A}}{\Phi} .}
\end{gathered}
$$

For the case that $\Phi$ is very small, the optimum step size is the step size which balances the truncation and condition errors [54. Typically one way to estimate $\epsilon_{A}$ is to monitor the "cost" functional after the desired residual values are reached (i.e. when the convergence criteria is met). Oscillations in sensitivity in steady state provide an estimation of $\epsilon_{A}$.

\section{References}

[1] B. A. Cipra, Energy-efficient building design, [Online; accessed 20-February-2017] (2013). URL https://sinews.siam.org/Details-Page/energy-efficient-building-design-1/

[2] Q. Chen, Ventilation performance prediction for buildings: A method overview and recent applications, Building and environment 44 (4) (2009) 848-858.

[3] W. Liu, T. Zhang, Y. Xue, Z. J. Zhai, J. Wang, Y. Wei, Q. Chen, State-of-the-art methods for inverse design of an enclosed environment, Building and Environment 91 (2015) 91-100.

[4] T. Zhang, Q. Chen, Identification of contaminant sources in enclosed environments by inverse CFD modeling, Indoor air 17 (3) (2007) 167-177. 
[5] T. Zhang, Q. Chen, Identification of contaminant sources in enclosed spaces by a single sensor, Indoor air 17 (6) (2007) 439-449.

[6] T. T. Zhang, S. Yin, S. Wang, An inverse method based on CFD to quantify the temporal release rate of a continuously released pollutant source, Atmospheric environment 77 (2013) 62-77.

[7] W. Liu, M. Jin, C. Chen, Q. Chen, Optimization of air supply location, size, and parameters in enclosed environments using a computational fluid dynamics-based adjoint method, Journal of Building Performance Simulation 9 (2) (2016) 149-161.

[8] A. Sempey, C. Inard, C. Ghiaus, C. Allery, Fast simulation of temperature distribution in air conditioned rooms by using proper orthogonal decomposition, Building and Environment 44 (2) (2009) 280-289.

[9] B. Kramer, P. Grover, P. Boufounos, M. Benosman, S. Nabi, Sparse sensing and DMD based identification of flow regimes and bifurcations in complex flows, arXiv preprint arXiv:1510.02831.

[10] Y. Xue, Z. J. Zhai, Q. Chen, Inverse prediction and optimization of flow control conditions for confined spaces using a CFD-based genetic algorithm, Building and Environment 64 (2013) 77-84.

[11] C. W. Rowley, S. T. Dawson, Model reduction for flow analysis and control, Annual Review of Fluid Mechanics 49 (2017) 387-417.

[12] W. K. Anderson, V. Venkatakrishnan, Aerodynamic design optimization on unstructured grids with a continuous adjoint formulation, Computers \& Fluids 28 (4) (1999) 443-480.

[13] F. Muyl, L. Dumas, V. Herbert, Hybrid method for aerodynamic shape optimization in automotive industry, Computers \& Fluids 33 (5) (2004) 849-858.

[14] R. Lanzafame, M. Messina, Fluid dynamics wind turbine design: Critical analysis, optimization and application of bem theory, Renewable energy 32 (14) (2007) 2291-2305.

[15] C. Othmer, A continuous adjoint formulation for the computation of topological and surface sensitivities of ducted flows, International Journal for Numerical Methods in Fluids 58 (8) (2008) 861-877.

[16] R. Kerswell, C. Pringle, A. Willis, An optimization approach for analysing nonlinear stability with transition to turbulence in fluids as an exemplar, Reports on Progress in Physics 77 (8) (2014) 085901.

[17] D. Foures, C. Caulfield, P. J. Schmid, Optimal mixing in two-dimensional plane poiseuille flow at finite péclet number, Journal of Fluid Mechanics 748 (2014) 241-277.

[18] S. Rabin, C. Caulfield, R. Kerswell, Designing a more nonlinearly stable laminar flow via boundary manipulation, Journal of Fluid Mechanics 738 (2014) R1.

[19] J. A. Burns, J. Borggaard, E. Cliff, L. Zietsman, An optimal control approach to sensor / actuator placement for optimal control of high performance buildings, International High Performance Buildings Conference at Purdue, July 16-19, 2012.

[20] X. Zhao, W. Liu, S. Liu, Y. Zou, Q. Chen, Inverse design of an indoor environment using a CFDbased adjoint method with the adaptive step size for adjusting the design parameters, Numerical Heat Transfer, Part A: Applications 71 (7) (2017) 707-720.

[21] J. Borggaard, J. A. Burns, A. Surana, L. Zietsman, Control, estimation and optimization of energy efficient buildings, in: American Control Conference, 2009. ACC'09., IEEE, 2009, pp. 837-841.

[22] W. Liu, Q. Chen, Optimal air distribution design in enclosed spaces using an adjoint method, Inverse Problems in Science and Engineering 23 (5) (2015) 760-779. 
[23] E. Turgeon, D. Pelletier, J. Borggaard, A continuous sensitivity equation approach to optimal design in mixed convection, Numerical Heat Transfer: Part A: Applications 38 (8) (2000) 869-885.

[24] P. F. Linden, The fluid mechanics of natural ventilation, Annual review of fluid mechanics 31 (1) (1999) 201-238.

[25] W. Baines, J. Turner, Turbulent buoyant convection from a source in a confined region, Journal of Fluid mechanics 37 (01) (1969) 51-80.

[26] P. Linden, G. Lane-Serff, D. Smeed, Emptying filling boxes: the fluid mechanics of natural ventilation, Journal of Fluid Mechanics 212 (1990) 309-335.

[27] N. Kaye, G. Hunt, Time-dependent flows in an emptying filling box, Journal of Fluid Mechanics 520 (2004) 135-156.

[28] D. Bolster, C. Caulfield, Transients in natural ventilationa time-periodically-varying source, Building Services Engineering Research and Technology 29 (2) (2008) 119-135.

[29] D. Bower, C. Caulfield, S. Fitzgerald, A. Woods, Transient ventilation dynamics following a change in strength of a point source of heat, Journal of Fluid Mechanics 614 (2008) 15.

[30] S. Nabi, M. Flynn, The hydraulics of exchange flow between adjacent confined building zones, Building and Environment 59 (2013) 76-90.

[31] J. Craske, M. van Reeuwijk, Generalised unsteady plume theory, Journal of Fluid Mechanics 792 (2016) $1013-1052$.

[32] B. Morton, G. Taylor, J. Turner, Turbulent gravitational convection from maintained and instantaneous sources, in: Proceedings of the Royal Society of London A: Mathematical, Physical and Engineering Sciences, Vol. 234, The Royal Society, 1956, pp. 1-23.

[33] S. Nabi, M. Flynn, Buoyancy-driven exchange flow between two adjacent building zones connected with top and bottom vents, Building and Environment 92 (2015) 278-291.

[34] B. Mohammadi, O. Pironneau, Analysis of the k-epsilon turbulence model (1993) France: Editions MASSON.

[35] R. Henkes, F. Van Der Vlugt, C. Hoogendoorn, Natural-convection flow in a square cavity calculated with low-reynolds-number turbulence models, International Journal of Heat and Mass Transfer 34 (2) (1991) 377-388.

[36] Openfoam - the open source computational fluid dynamics (cfd) toolbox (Dec. 2016). URL http://openfoam.com

[37] H. G. Weller, G. Tabor, H. Jasak, C. Fureby, A tensorial approach to computational continuum mechanics using object-oriented techniques, Computers in physics 12 (6) (1998) 620-631.

[38] S. V. Patankar, D. B. Spalding, A calculation procedure for heat, mass and momentum transfer in three-dimensional parabolic flows, International journal of heat and mass transfer 15 (10) (1972) 17871806.

[39] P. K. Sweby, High resolution schemes using flux limiters for hyperbolic conservation laws, SIAM journal on numerical analysis 21 (5) (1984) 995-1011.

[40] B. Van Leer, Towards the ultimate conservative difference scheme. ii. monotonicity and conservation combined in a second-order scheme, Journal of computational physics 14 (4) (1974) 361-370.

[41] J. H. Ferziger, M. Peric, A. Leonard, Computational methods for fluid dynamics (1997). 
[42] N. Kaye, G. Hunt, The effect of floor heat source area on the induced airflow in a room, Building and Environment 45 (4) (2010) 839-847.

[43] G. Hunt, H. Burridge, Fountains in industry and nature, Annual Review of Fluid Mechanics 47 (2015) $195-220$.

[44] P. O. Fanger, et al., Thermal comfort. analysis and applications in environmental engineering., Thermal comfort. Analysis and applications in environmental engineering.

[45] E. Papoutsis-Kiachagias, K. Giannakoglou, Continuous adjoint methods for turbulent flows, applied to shape and topology optimization: industrial applications, Archives of Computational Methods in Engineering 23 (2) (2016) 255-299.

[46] D. Foures, C. Caulfield, P. Schmid, Variational framework for flow optimization using seminorm constraints, Physical Review E 86 (2) (2012) 026306.

[47] S. Boyd, L. Vandenberghe, Convex optimization, Cambridge university press, 2004.

[48] ASHRAE Standard 55-1992, Thermal environmental conditions for human occupancy, 1992.

[49] N. Kaye, Y. Ji, M. Cook, Numerical simulation of transient flow development in a naturally ventilated room, Building and Environment 44 (5) (2009) 889-897.

[50] A. Novoselac, J. Srebric, A critical review on the performance and design of combined cooled ceiling and displacement ventilation systems, Energy and buildings 34 (5) (2002) 497-509.

[51] D. L. Loveday, K. C. Parsons, A. H. Taki, S. G. Hodder, et al., Designing for thermal comfort in combined chilled ceiling/displacement ventilation environments/discussion, ASHRAE transactions 104 (1998) 901.

[52] E. Laftchiev, D. Nikovski, An iot system to estimate personal thermal comfort, in: Internet of Things (WF-IoT), 2016 IEEE 3rd World Forum on, IEEE, 2016, pp. 672-677.

[53] F. Tröltzsch, Optimal control of partial differential equations, volume 112 of graduate studies in mathematics, American Mathematical Society, Providence, RI.

[54] J. Iott, R. T. Haftka, H. M. Adelman, Selecting step sizes in sensitivity analysis by finite differences, NASA Technical Memorandum, 1985. 